Research Paper

\title{
NADPH oxidase 4 (Nox4) deletion accelerates liver regeneration in mice
}

\author{
M. Herranz-Itúrbide ${ }^{\text {a,b }}$, J. López-Luque ${ }^{\text {a,b }}$, E. Gonzalez-Sanchez ${ }^{\text {a,b,c }}$, D. Caballero-Díaz ${ }^{\text {a,b }}$,

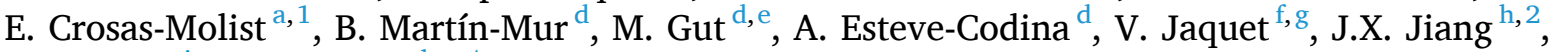 \\ N.J. Török ${ }^{\mathrm{i}}$, I. Fabregat ${ }^{\mathrm{a}, \mathrm{b}, \mathrm{c}, ",}$ \\ ${ }^{\text {a }}$ TGF- $\beta$ and Cancer Group. Oncobell Program, Bellvitge Biomedical Research Institute (IDIBELL), L'Hospitalet de Llobregat, Barcelona, Spain \\ ${ }^{\mathrm{b}}$ Oncology Program, CIBEREHD, National Biomedical Research Institute on Liver and Gastrointestinal Diseases, Instituto de Salud Carlos III, Spain \\ ${ }^{\mathrm{c}}$ Department of Physiological Sciences, Faculty of Medicine and Health Sciences, University of Barcelona, Spain \\ d CNAG-CRG, Centre for Genomic Regulation, Barcelona Institute of Science and Technology, Barcelona, Spain \\ ${ }^{\mathrm{e}}$ Universitat Pompeu Fabra, Barcelona, Spain \\ ${ }^{\mathrm{f}}$ Department of Pathology and Immunology, Medical School, University of Geneva, Geneva, Switzerland \\ ${ }^{\mathrm{g}}$ RE.A.D.S Unit, Medical School, University of Geneva, Geneva, Switzerland \\ ${ }^{\mathrm{h}}$ Gastroenterology and Hepatology, UC Davis, Sacramento, CA, USA \\ ${ }^{\mathrm{i}}$ Department of Internal Medicine, Division of Gastroenterology and Hepatology, Stanford University, Stanford, CA, USA
}

\section{A R T I C L E I N F O}

\section{Keywords:}

NADPH oxidase

NOX4

Liver regeneration

Hepatectomy

MYC

TGF-BETA

\begin{abstract}
A B S T R A C T
Liver is a unique organ in displaying a reparative and regenerative response after acute/chronic damage or partial hepatectomy, when all the cell types must proliferate to re-establish the liver mass. The NADPH oxidase NOX4 mediates Transforming Growth Factor-beta (TGF- $\beta$ ) actions, including apoptosis in hepatocytes and activation of stellate cells to myofibroblasts. Aim of this work was to analyze the impact of NOX4 in liver regeneration by using two mouse models where Nox4 was deleted: 1) general deletion of Nox4 (NOX4-/-) and 2) hepatocyte-specific deletion of Nox4 (NOX4hepKO). Liver regeneration was analyzed after $2 / 3$ partial hepatectomy $(\mathrm{PH})$. Results indicated an earlier recovery of the liver-to-body weight ratio in both NOX4-/- and NOX4hepKO mice and an increased survival, when compared to corresponding WT mice. The regenerative hepatocellular fat accumulation and the parenchyma organization recovered faster in NOX4 deleted livers. Hepatocyte proliferation, analyzed by Ki67 and phospho-Histone3 immunohistochemistry, was accelerated and increased in NOX4 deleted mice, coincident with an earlier and increased Myc expression. Primary hepatocytes isolated from NOX4 deleted mice showed higher proliferative capacity and increased expression of $M y c$ and different cyclins in response to serum. Transcriptomic analysis through RNA-seq revealed significant changes after PH in NOX4-/- mice and support a relevant role for $M y c$ in a node of regulation of proliferation-related genes. Interestingly, RNA-seq also revealed changes in the expression of genes related to activation of the TGF- $\beta$ pathway. In fact, levels of active TGF- $\beta 1$, phosphorylation of Smads and levels of its target p21 were lower at $24 \mathrm{~h}$ in NOX4 deleted mice. Nox4 did not appear to be essential for the termination of liver regeneration in vivo, neither for the in vitro hepatocyte response to TGF- $\beta 1$ in terms of growth inhibition, which suggest its potential as therapeutic target to improve liver regeneration, without adverse effects.
\end{abstract}

\section{Introduction}

The NADPH oxidase (NOX) family has recently emerged as an important source of reactive oxygen species (ROS), which play relevant roles in signal transduction under both physiological and pathological situations [1]. The NOX4 isoform shows unique characteristics compared to other NOXes [2], since it produces large amounts of hydrogen peroxide $\left(\mathrm{H}_{2} \mathrm{O}_{2}\right)$ constitutively, does not require GTPase Rac

\footnotetext{
Abbreviations: EGF, Epidermal growth factor; EGFR, Epidermal growth factor receptor; HCC, Hepatocellular carcinoma; HGF, Hepatocyte growth factor; HC, Hydrocortisone; INS, Insulin; Nox, NADPH oxidase; PH, Partial hepatectomy; ROS, Reactive oxygen species; TGF- $\beta$, Transforming Growth Factor-beta.

* Corresponding author. IDIBELL, Gran Vía de l'Hospitalet, 199, 08908, L'Hospitalet de Llobregat, Barcelona, Spain.

E-mail address: ifabregat@idibell.cat (I. Fabregat).

1 Current address: Barts Cancer Institute, Queen Mary University of London, John Vane Science Building, Charterhouse Square, London, EC1M 6BQ, UK.

2 Current address: Department of Molecular Biosciences, School of Veterinary Medicine, UC Davis, Sacramento, CA, USA.
} 
for its activity and it is associated not only to cell membrane, but also to internal membranes, where $\mathrm{H}_{2} \mathrm{O}_{2}$ generation occurs [3]. In the liver, NOX4 plays relevant roles mediating Transforming Growth Factor-beta (TGF- $\beta$ ) actions in fibrosis and hepatocarcinogenesis [4,5]. We previously described that NOX 4 mediates TGF- $\beta 1$-induced myofibroblast activation, contributing to the development of liver fibrosis [6,7]. Indeed, there is an increasing interest in the development of NOX4 inhibitors as therapeutic tools for chronic liver diseases [8]. However, in hepatocytes and liver tumor cells, we found that NOX4 mediates TGF- $\beta 1$-induced tumor suppressor actions, particularly apoptosis [9]. Furthermore, when NOX4 expression is knocked-down in hepatocellular carcinoma (HCC) cells, they acquire higher proliferative and migratory capacity in vitro and higher tumorigenic potential in in vivo xenografts in nude mice $[10,11]$. Interestingly, NOX4 gene deletions were found in HCC patients, which correlate with a decrease in NOX4 protein levels [11].

Liver regeneration is the response to loss of hepatic tissue, which may occur as a result of toxic injury, exposure to infection, traumatic damage or surgical resection [12]. Hepatocytes are the major functional cells of the liver. Indeed, in most cases, the regenerative response is strongly triggered when there is a loss of hepatocytes at a large scale. Many genes are involved in liver regeneration, but the essential circuitry for the process involves the participation of cytokines, growth factors and metabolic networks [13]. Under normal conditions, all hepatic cells undergo one to three rounds of replication, but evidence strongly suggests that under conditions where the proliferation of hepatocytes or biliary cells is inhibited, they can act as facultative stem cells for each other and replenish the inhibited cellular compartment by a process of transdifferentiation [14]. Liver cell proliferation occurs also at any time in normal liver, although very few hepatocytes proliferate and the mechanisms involved in this slow process in the absence of liver injury are not well understood [15]. Partial hepatectomy is one of the most studied animal experimental models of liver regeneration [16]. The complexity of the signaling pathways initiating and terminating this process has provided paradigms for regenerative medicine and any aspect of the mechanisms involved continues to be under active investigation.

Very little is known about the role of NOXes during liver regeneration. However, considering the function of NOX4 in inhibiting hepatocyte proliferation, we previously reported that Nox4 expression is downregulated after partial hepatectomy in mice [10]. The aim of this work was to understand more about the role of Nox4 in this process taking advantage of experimental mouse models were Nox4 was deleted either in all cells or specifically in hepatocytes. The study is not only of interest because it provides an advanced understanding of the molecular mechanisms that regulate liver regeneration, but also to anticipate the potential of NOX4 inhibitors as therapeutic tools in human chronic liver pathologies.

\section{Material and methods}

\subsection{Animal models}

NOX4-/- (B6.129-Nox4tm1Kkr/J) mice, generated in Dr. Krause's Laboratory [17], were obtained from Jackson Laboratories (together with the corresponding C57BL/6J wild type (WT) mice) and housed at IDIBELL (Barcelona, Spain). Nox4 hepatocyte-specific knockout (NOX4hepKO) mice on a C57BL/6J background were generated in Dr. Török's laboratory [18] and housed at University of California Davis (Sacramento, CA, USA). See Supplementary Fig. 1A for schematic description of the mouse models. 8 to 16 -week-old male and female mice, hosted under $12 \mathrm{~h}$ light/dark cycle with free access to food and water were used in the study. All experiments complied with the EU Directive 2010/63/UE for animal experiments and the institution's guidelines (Ethical Committee for animal experimentation of IDIBELL) and were approved by the General Direction of Environment and
Biodiversity, Government of Catalonia (experiments in NOX4-/- mice, \#4589) and the Animal Care Committee of UC Davis (experiments in NOX4hepKO mice).

\subsection{Experimental model of liver regeneration}

2/3 partial Hepatectomy ( $\mathrm{PH}$ ) was performed as described by Higgins and Anderson [19]. Mice were euthanized at different times after surgery, as indicated in the figures. Tissue samples were immediately frozen in liquid nitrogen, cryopreserved in optimal cutting temperature compound (OCT) or fixed in $4 \%$ paraformaldehyde (PFA) and paraffin-embedded for further analysis. SHAM operated mice were used as control. Number of animals used in the study were minimized for ethical reasons. Thus, for NOX4-/- model between 3-10 animals were used per timepoint. For NOX4hepKO model between 2-3 animals per timepoint were analyzed and 2 different RNA extractions per animal liver tissue were done.

\subsection{Isolation of primary hepatocytes, cell culture and treatments}

Primary hepatocytes from NOX4hepKO, NOX4-/- and the corresponding WT mice were isolated by collagenase (C5138, Sigma-Aldrich, St. Louis, MO) in situ perfusion as previously described [20], then cultured on collagen-coated plates using Williams'E medium (W4125, Sigma-Aldrich, St. Louis, MO, USA) supplemented with 10-15\% Fetal Bovine Serum (FBS), Penicillin (100 U/ml), Streptomycin $(100 \mu \mathrm{g} / \mathrm{mL})$ and maintained in a humidified atmosphere of $37^{\circ} \mathrm{C}, 5 \% \mathrm{CO}_{2}$. Between 20-36 h after isolation, cells were serum-starved for 4-8 $\mathrm{h}$ before incubation with fresh Williams'E medium supplemented with: FBS ( $2 \%$ or $15 \%)$, human recombinant TGF- $\beta 1$ ( $2 \mathrm{ng} / \mathrm{mL})$ (616455, Calbiochem, La Jolla, CA, USA), Insulin ( $2 \mu \mathrm{M})$ (I9278, Sigma-Aldrich, St. Louis, MO, USA), Hydrocortisone $(100 \mu \mathrm{M})$ (H2270, Sigma-Aldrich, St. Louis, MO, USA), EGF (20 ng/mL) (E9644, Sigma-Aldrich, St. Louis, MO, USA), as indicated in the figures.

\subsection{Western blot analysis}

Protein extracts and western blotting procedures were carried out as previously described [21]. Briefly, samples were lysed in RIPA lysis buffer supplemented with a protease inhibitors cocktail (1 mM PMSF, $5 \mu \mathrm{g} / \mathrm{mL}$ Leupeptin, $0.1 \mathrm{mM} \mathrm{Na} \mathrm{VO}_{4}, 0.5 \mathrm{mM}$ DTT, $20 \mathrm{mM} \beta$-Glycerolphosphate) for $1 \mathrm{~h}$ at $4{ }^{\circ} \mathrm{C}$. Protein was quantified with the Bio-Rad Protein Assay Dye Reagent Concentrate (Bio-Rad Laboratories, USA). Primary antibodies are summarized in Supplementary Table I. ECL Mouse IgG and Rabbit IgG, HRP-Linked antibodies (GE Healthcare, Buckinghamshire, UK) were used at 1:2000. Densitometric analysis was performed using ImageJ software (National Institutes of Health [NIH], Bethesda, MD, USA).

\subsection{Analysis of cell viability}

Viable cell numbers were determined by crystal violet staining. Cells were plated in 24-well plates. After treatments, culture medium was removed, cells were washed twice with PBS and then stained with crystal violet $(0.2 \% \mathrm{w} / \mathrm{v}$ in $2 \%$ ethanol) for $30 \mathrm{~min}$. Next, staining solution was removed, and cells were washed with PBS to remove dye excess before being lysed in 10\% Sodium Dodecyl Sulphate (SDS) for $30 \mathrm{~min}$. Absorbance was measured at $595 \mathrm{~nm}$. Results were then calculated as the percentage of viable cells relative to time zero.

\subsection{Immunofluorescence staining}

For fluorescence microscopy studies, cells were plated on gelatincoated glass coverslips. Primary hepatocytes were fixed with $4 \%$ PFA and permeabilized during $2 \mathrm{~min}$ with $0.1 \%$ Triton X-100. Cells were incubated with the primary antibody (Supplementary Table I) during 
$1 \mathrm{~h}$, and then with a secondary anti-rabbit antibody coupled to Alexa Fluor 488 (Molecular Probes, Eugene, OR, USA) for $1 \mathrm{~h}$. Nuclei were stained using DAPI (Sigma-Aldrich, Merck, Madrid, Spain). Cells were visualized in a Nikon eclipse $80 \mathrm{i}$ microscope and in a Leica TSC SL spectral confocal microscope. Representative images were taken and edited in Adobe Photoshop. ImageJ software (National Institutes of Health [NIH], Bethesda, MD, USA) was used to quantify positive nuclei.

\subsection{Immunohistochemistry and histology analysis}

Paraffin-embedded tissues were cut into 4 - $\mu \mathrm{m}$-thick sections. Hematoxylin and Eosin (H\&E) staining and immunohistochemistry (IHC) analysis were performed using standard procedures [21]. For IHC assays, sections were incubated overnight at $4{ }^{\circ} \mathrm{C}$ with the corresponding primary antibodies. Binding was developed with the Vectastain ABC KIT rabbit or mouse (PK-4001 and PK-4002, Vector Laboratories Inc., Burlingame, CA, USA). Antibodies used and conditions are summarized in Supplementary Table I. Tissues were visualized in a Nikon Eclipse 80i microscope and representative images were taken with a Nikon DS-Ri1 digital camera. ImageJ software (National Institutes of Health [NIH], Bethesda, MD, USA) was used to quantify all the parameters.

\subsection{Histological analysis of hepatic lipids}

Hepatic lipid drops were analyzed using Oil Red O staining. 10- $\mu \mathrm{m}-$ thick tissue sections in OCT were sequentially fixed with 4\% PFA, washed with distilled water, rinsed with $60 \%$ isopropanol and washed with distilled water again. Then tissues were stained with freshly prepared Oil Red O (O1391, Sigma Aldrich, St. Louis, MO, USA) working solution (3:2 $\mathrm{v} / \mathrm{v}$ in distilled water) for $10 \mathrm{~min}$ at room temperature. After rinsing with $60 \%$ isopropanol and washing with distilled water for $5 \mathrm{~min}$, tissues were stained with Hematoxylin and mounted in Mowiol. Lipid droplets were visualized with a Nikon eclipse $80 \mathrm{i}$ microscope and representative images were taken with a Nikon DS-Ri1 digital camera.

\subsection{Analysis of gene expression}

E.Z.N.A. ${ }^{\circledR}$ Total RNA Kit II (Omega bio-tek, Norcross, GA, USA) was used following manufacturer's protocol for total RNA isolation as previously described for tissues [21] and cells [22]. Reverse transcription (RT) was carried out with random primers using High Capacity RNA to cDNA Master Mix Kit (Applied Biosystems, Foster City, CA, USA). $1 \mu \mathrm{g}$ of total RNA per sample was used. mRNA expression levels were determined in duplicates in a LightCycler ${ }^{\circledR} 480$ Real Time PCR System, using the LightCycler ${ }^{\circledR} 480$ SYBR Green I Master Mix (Roche Diagnostics GmbH, Mannheim, Germany). Gene expression was normalized to Rpl32 mRNA content. See Supplementary Table II for primers sequences.

\subsection{RNA sequencing (RNA-seq)}

Total RNA from Mus musculus was quantified by Qubit ${ }^{\circledR}$ RNA BR Assay kit (Thermo Fisher Scientific) and the RNA integrity was estimated by using RNA 6000 Nano Bioanalyzer 2100 Assay (Agilent). The RNA-seq libraries were prepared with KAPA Stranded mRNA-Seq Illumina ${ }^{\circledR}$ Platforms Kit (Roche) following the manufacturer's recommendations. Briefly, $500 \mathrm{ng}$ of total RNA was used for the poly-A fraction enrichment with oligo-dT magnetic beads, following the mRNA fragmentation. The strand specificity was achieved during the second strand synthesis performed in the presence of dUTP instead of dTTP. The bluntended double stranded cDNA was 3'adenylated and Illumina platform compatible adaptors with unique dual indexes and unique molecular identifiers (Integrated DNA Technologies) were ligated. The ligation product was enriched with 15 PCR cycles and the final library was validated on an Agilent 2100 Bioanalyzer with the DNA 7500 assay. The libraries were sequenced on HiSeq 4000 (Illumina) with a read length of $2 \mathrm{x} 51 \mathrm{bp}+17 \mathrm{bp}+8 \mathrm{bp}$ using HiSeq 4000 SBS kit (Illumina) and HiSeq
4000 PE Cluster kit (Illumina), following the manufacturer's protocol for dual indexing. Image analysis, base calling and quality scoring of the run were processed using the manufacturer's software Real Time Analysis (RTA 2.7.7).

Reads obtained by RNA-seq were mapped against Mus musculus reference genome (GRCm38) using STAR software version 2.5.3a [23] with ENCODE parameters for long reads. Annotated genes were quantified using RSEM version 1.3.0 [24] with default parameters and the annotation file from GENCODE version M15. Differential expression analysis was performed withDESeq2 v1.26.0 R package [25], using a Wald test to compare the different time points between samples WT and NOX4-/-, and adjusting for sex. We considered differentially expressed genes (DEG) those with $\mathrm{p}$-value adjusted $<0.05$ and absolute fold-change $|\mathrm{FC}|>1.5$. The heatmap was plotted with the 'pheatmap' $R$ package using the shrunken log2 fold change of the DEG in each time point. Time course gene expression plots were generated using the normalized counts obtained with DESeq2 and the R package 'ggplot2'. A Gene Ontology (GO) term enrichment analysis was performed with the differentially expressed genes using the R package gProfileR v0.7.0 [26], based on GO terms from the ENSEMBL database. Networks of protein-protein interactions were generated using the webtool STRINGdb (https://string-db.org/).

\subsection{Statistical analyses}

Data are represented as Mean \pm Standard Error of the Mean (SEM). Differences between groups were compared using Student's t-test with Welch correction (when comparing two groups) or Two-way ANOVA with Tukey's multiple comparison post-hoc test (differences between groups considering two independent variables). Survival curves were estimated by Kaplan-Meier analysis, and significance was tested by the log-rank test. Statistical calculations were performed using GraphPad Prism software (GraphPad for Science Inc., San Diego, CA, USA). Differences were considered statistically significant when $\mathrm{p}<0.05$.

\section{Results}

\subsection{Nox4 deletion accelerated liver mass recovery and parenchymal structure}

NOX4-/- and NOX4hepKO mice (see Material \& Methods section) were used to analyze the response to $2 / 3 \mathrm{PH}$. We previously reported that Nox4 expression decreases after PH in C57BL/6 mice [10]. Here we confirmed that decrease in Nox4 mRNA levels also occurred in NOX4floxp $+/+$ mice, the WT mice used for breeding and obtaining the NOX4hepKO mice (Supplementary Fig. 1B). We next analyzed how Nox4 deletion affects the liver expression of other members of the Nox gene family. Nox2 expression was not significantly different between WT and KO mice in any of the groups and its expression decreased, recovering later, in response to $\mathrm{PH}$ (no significant differences between WT and KO mice, Supplementary Fig. 1C). Expression of Nox1 was very low, barely detected by Real-Time PCR and with huge fluctuations among animals (Supplementary Fig. 1D). These results indicate that the absence of Nox4 expression is not compensated by differences in the expression of other members of the family.

When the Liver/Body weight ratio was analyzed at different times after $\mathrm{PH}$, we observed a significant earlier recovery in both NOX4-/and NOX4hepKO mice when compared to the corresponding WT mice (Fig. 1A and Supplementary Fig. 2A), which correlated with significant higher survival after $\mathrm{PH}$ in NOX4-/- mice (Fig. 1B). Although number of animals was lower to obtain statistical significance, a similar tendency was observed in NOX4hepKO mice (Supplementary Fig. 2B). Kinetics of the recovery after $\mathrm{PH}$ varied in both models of animals, probably due to the different background and different environment in the animal room (NOX4-/- mice were at IDIBELL in Barcelona; NOX4HepKO mice were at UC Davis, in CA). But it was clear that deletion of NOX4, either in the 
A

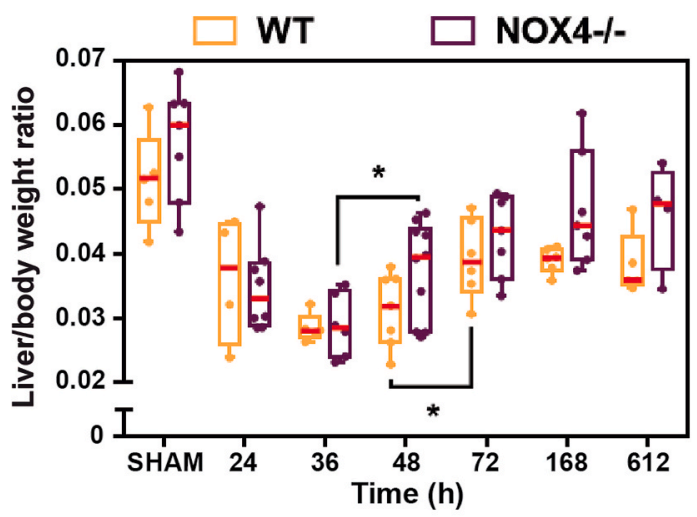

B

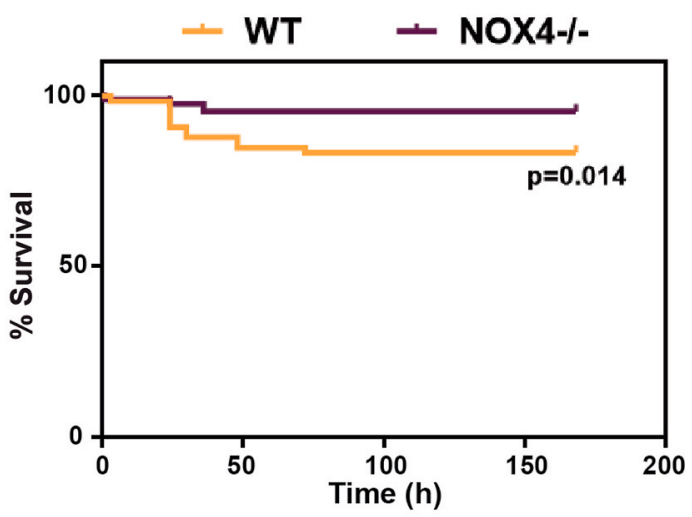

C

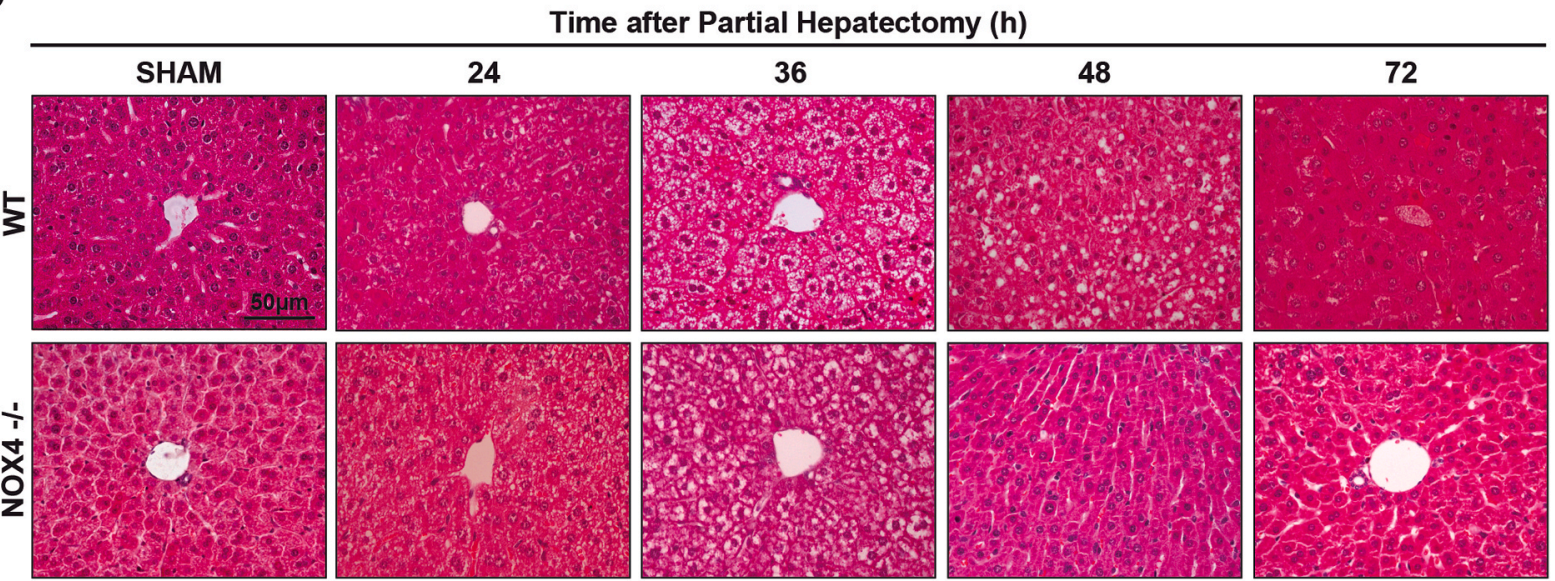

D

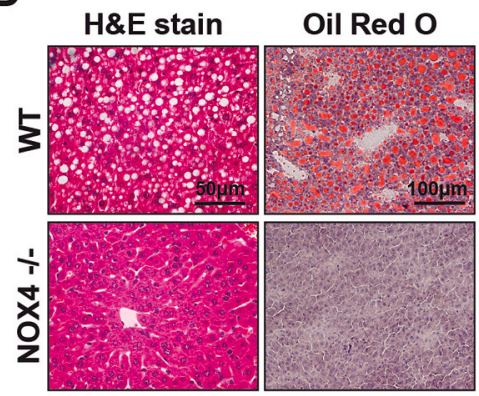

E

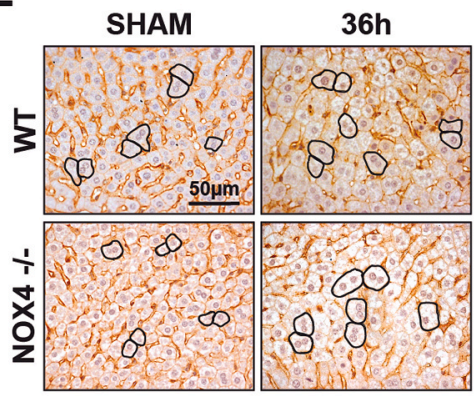

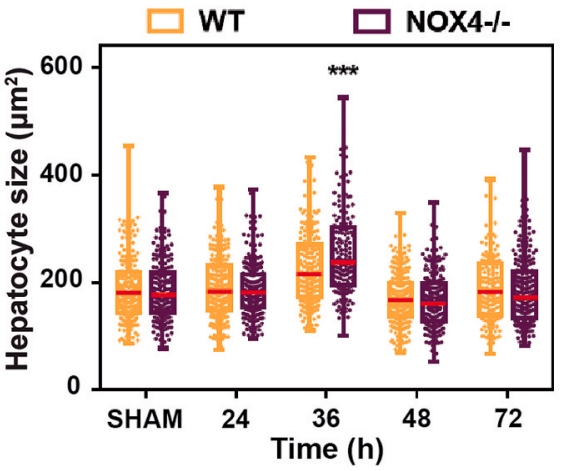

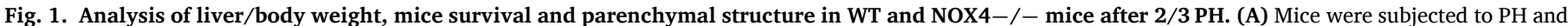

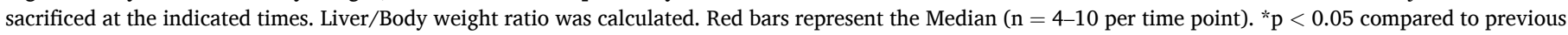

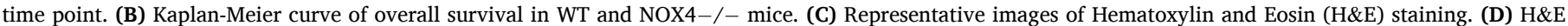

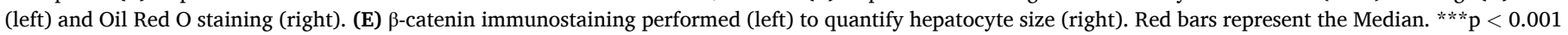
compared to WT. (For interpretation of the references to colour in this figure legend, the reader is referred to the Web version of this article.)

whole animal or in the hepatocytes, induced an earlier recovery of the liver mass. H\&E staining also revealed an earlier recovery of the parenchymal structure (Fig. 1C and Supplementary Fig. 2C). Liver steatosis, characteristic of the final G2/M phase of the hepatocyte cell cycle [12] was already resolved after $48 \mathrm{~h}$ in NOX4-/- mice, when maximal in WT yet (Fig. 1D). Furthermore, it was interesting to observe a transient higher size at $36 \mathrm{~h}$ after $\mathrm{PH}$ in hepatocytes from NOX4-/mice when compared to WT animals, which was also noticed in NOX4hepKO at 24 and $72 \mathrm{~h}$ after $\mathrm{PH}$, when compared to NOX4floxp $+/+$ (Fig. 1E and Supplementary Fig. 2D).

Altogether these results indicate that NOX4-/- and NOX4hepKO livers, after $2 / 3 \mathrm{PH}$, showed an earlier recovery of the liver parenchyma structure and regenerative steatosis, concomitant with a transient increase in hepatocyte size. Similar results were obtained in both NOX4-/- and NOX4HepKO mice and this would indicate that Nox4 silencing has a higher impact on the hepatocyte responses to $\mathrm{PH}$, which could be expected considering that the hepatocyte is the liver cell where, under normal physiological conditions, Nox4 expression is notably higher [6].

\subsection{Accelerated and increased hepatocyte proliferation in NOX4hepKO} mice after $2 / 3 \mathrm{PH}$

In order to analyze whether the proliferative response of the hepatocytes was altered in the absence of Nox4 expression, we performed IHC analysis of Ki67, as a marker of a proliferative cell cycle, and 
phospho-Histone3, as a more specific marker of G2/M phase, in the NOX4hepKO model. Results revealed a significant increase in the number of stained hepatocytes at 48 and $72 \mathrm{~h}$ after PH in NOX4hepKO when compared to NOX4floxp+/+ (Fig. 2A-B). Furthermore, NOX4hepKO hepatocytes reached the maximal DNA synthesis at $48 \mathrm{~h}$ after $\mathrm{PH}$, whereas NOX4floxp $+/+$ reached it at $72 \mathrm{~h}$.

With the aim of better exploring the proliferative capacity of the hepatocytes, we isolated hepatocytes from unhepatectomized mice and cultured them to evaluate the response to FBS. Interestingly, very low concentrations of FBS (2\%) allowed NOX4hepKO cells to increase almost $40 \%$ the number of viable cells in $24 \mathrm{~h}$, significantly higher than the increase observed in NOX4floxp $+/+$ hepatocytes (Fig. 2C). This increase correlated with a much higher increase in the expression of Cyclin D1 (Ccnd1), Cyclin A2 (Ccnd2) and Cyclin B2 (Ccnb2) (Fig. 2D). Similar results were obtained in NOX4-/- isolated primary hepatocytes, correlating with increased Ki67 positive nuclei (Supplementary Figs. 3A-C).
These results indicate that the absence of Nox4 expression primes hepatocytes for a faster and higher response to mitogenic signals.

\subsection{Accelerated regeneration correlated with an earlier induction of Myc expression}

We had previously described that $M y c$ expression is required for an efficient liver regeneration in mice [27], as its deletion delays the recovery of the liver mass. Livers from NOX4 $-/-$ mice showed a higher increase in Myc expression $24 \mathrm{~h}$ after $\mathrm{PH}$, and both 24 and $36 \mathrm{~h}$ after $\mathrm{PH}$ in NOX4HepKO livers (Fig. 3A) when compared to the corresponding WT mice. Western blot and IHC analysis revealed significantly higher c-Myc protein levels and c-Myc positive nuclei $36 \mathrm{~h}$ after PH in NOX4hepKO hepatocytes, which confirmed the differences observed at the mRNA level (Fig. 3B-C). Interestingly, primary cultures of hepatocytes from WT and NOX4-/- mice $30 \mathrm{~h}$ after $\mathrm{PH}$, showed much higher increase in Myc mRNA levels in NOX4-/- hepatocytes in response to FBS

A

Time After Partial Hepatectomy (h)
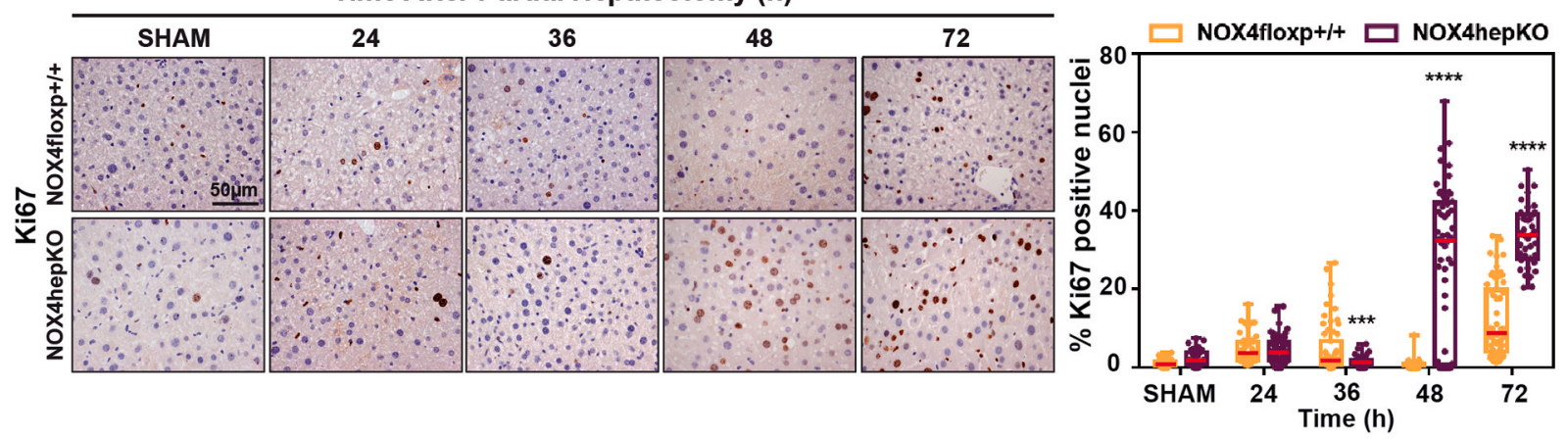

B
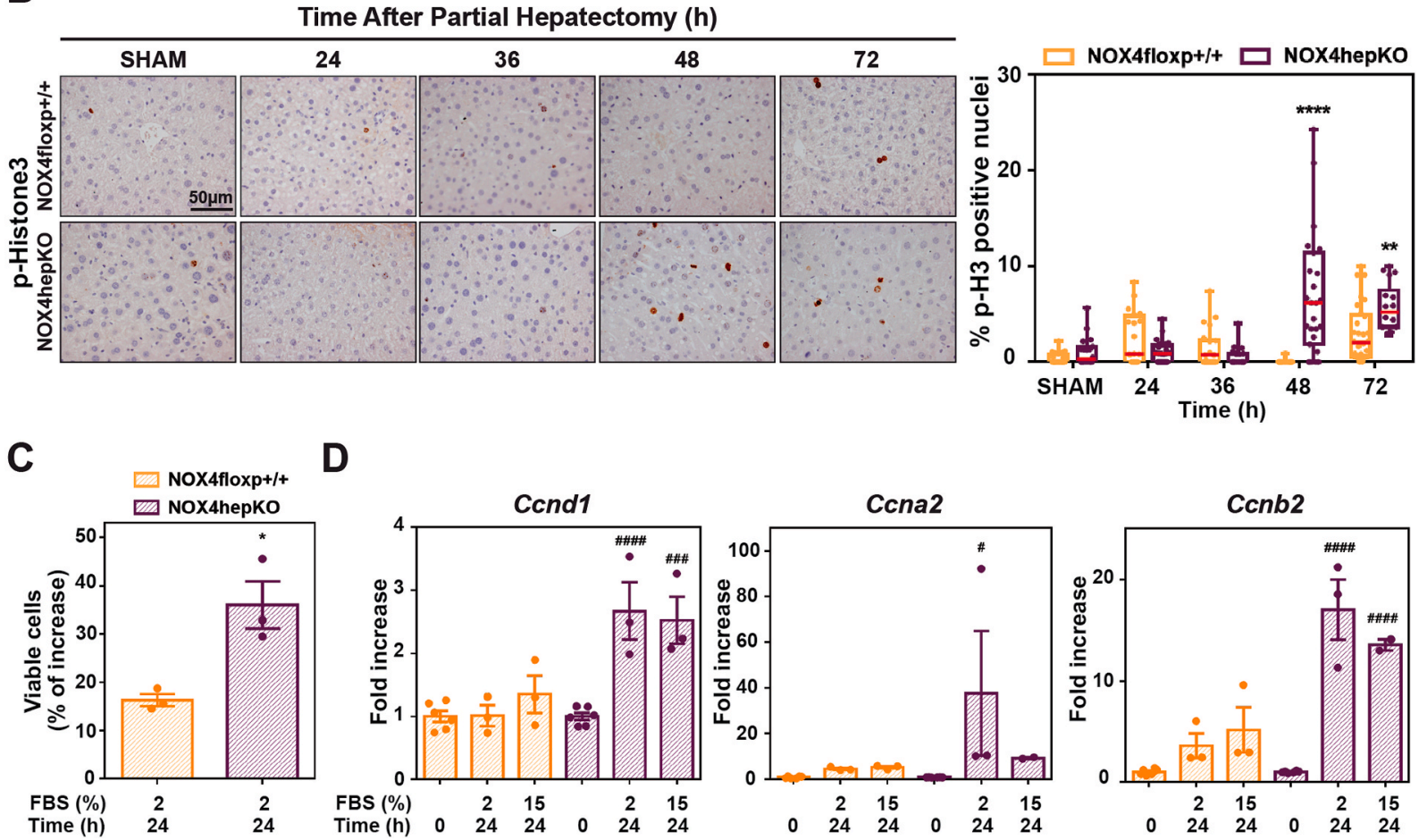

Fig. 2. Hepatocyte proliferation in NOX4floxp $+/+$ and NOX4hepKO analyzed in livers after PH and in cultured hepatocytes. (A) Ki67 and (B) p-Histone 3 immunostaining was performed in NOX4floxp $+/+$ and NOX4hepKO mice (left) and positive nuclei were quantified with ImageJ software (right). Red bars represent the Median. $* * \mathrm{p}<0.01, * * * \mathrm{p}<0.001, * * * * \mathrm{p}<0.0001$ compared to NOX4floxp $+/+$. (C-D) Primary hepatocytes isolated from NOX4floxp $+/+$ and NOX4hepKO mice were treated as indicated in the graph: C: Viable cell number represented as \% of increase versus 0 time; D: RT-qPCR analysis of Ccnd1, Ccna2 and Ccnb2. Relative expression to Rpl32 gene. In C and D, Mean \pm SEM $(\mathrm{n}=3)$. ${ }^{*} \mathrm{p}<0.05$ compared to NOX4floxp $+/+(C)$. \#p $<0.05$, \#\#\#p $<0.001$, \#\#\#\#p $<0.0001$ compared to untreated cells (D). (For interpretation of the references to colour in this figure legend, the reader is referred to the Web version of this article.) 
A

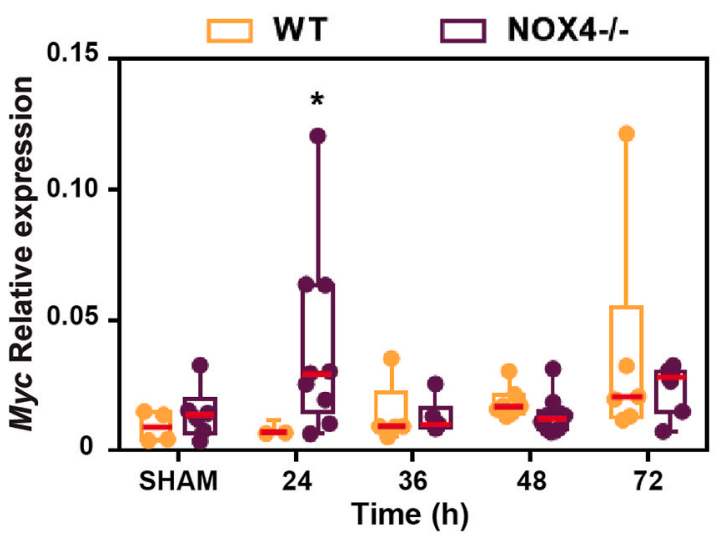

B
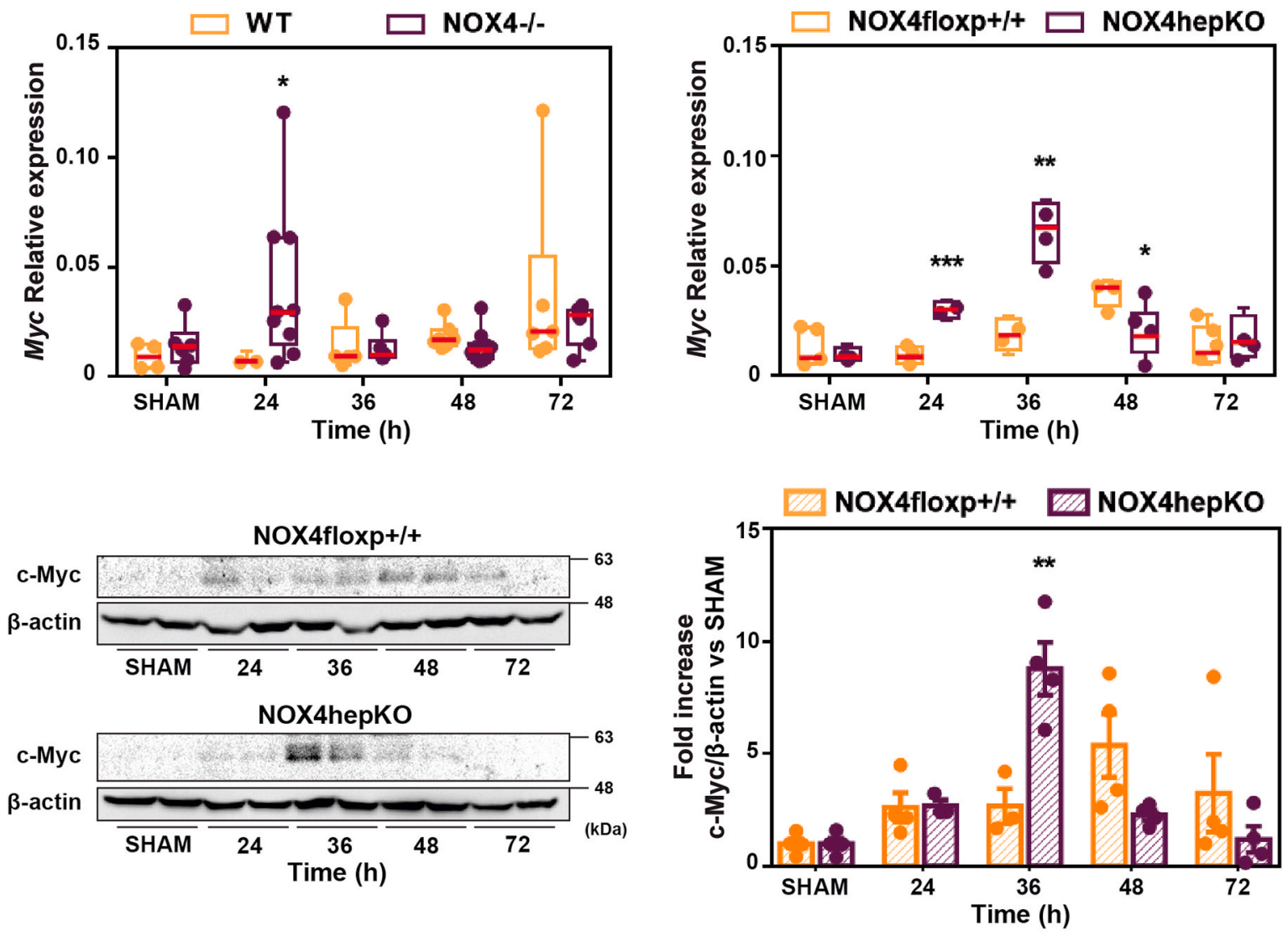

C
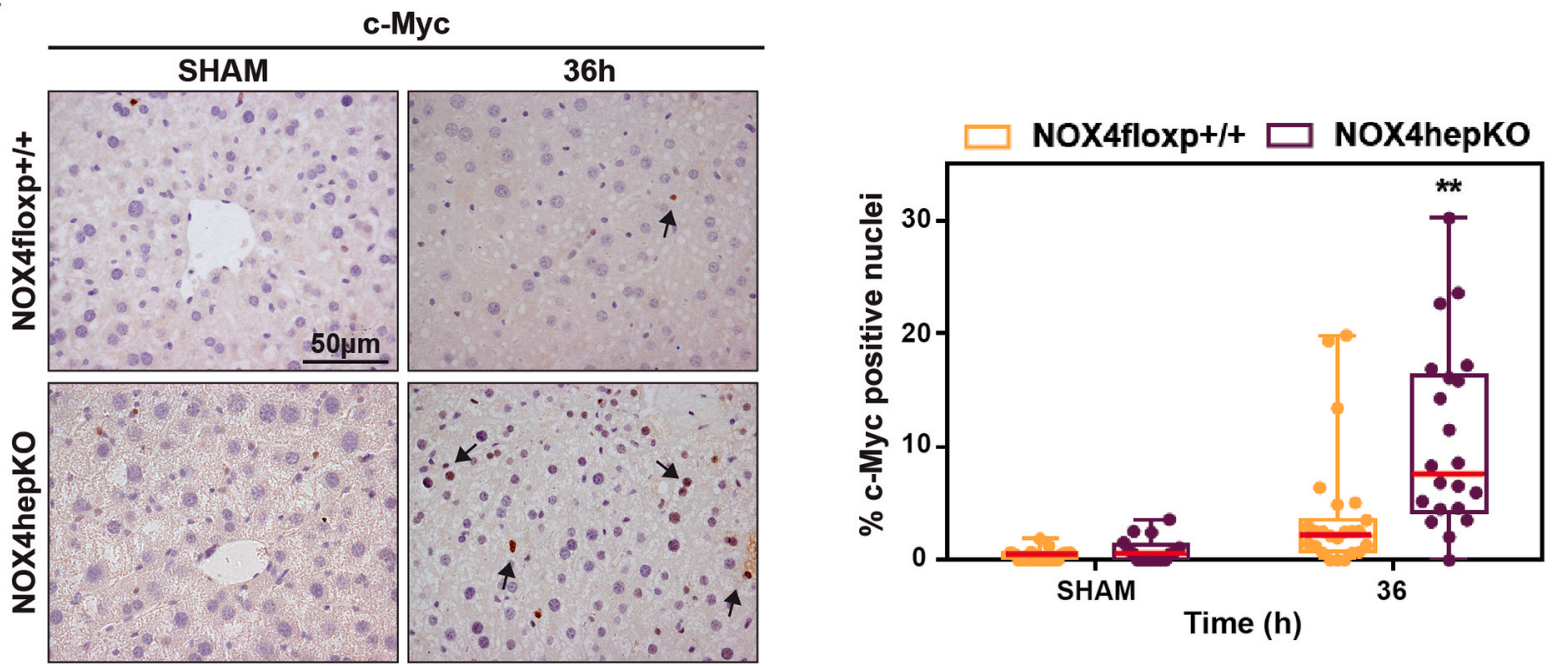

Fig. 3. Analysis of c-Myc expression in livers after PH. (A) RT-qPCR analysis of Myc in livers from NOX4-/- and NOX4hepKO mice and their respective WT controls. Relative expression to Rpl32 gene. Red bars represent the Median ( $n=4-10$ per time point in NOX4-/- model; $n=4-6$ per time point in NOX4hepKO model). *p $<0,05,{ }^{* *} \mathrm{p}<0.01, * * * \mathrm{p}<0.001$, compared to WT controls. (B) Analysis of c-Myc by Western Blot in NOX4floxp $+/+$ and NOX4hepKO livers (left) and densitometry of the different experiments performed (right): Mean \pm SEM $(n=3-4$ per time point). $\beta$-actin was used as a loading control. (C) c-Myc immunostaining in NOX4floxp $+/+$ and NOX4hepKO livers after $36 \mathrm{~h} \mathrm{PH} \mathrm{(left);} \mathrm{positive} \mathrm{nuclei} \mathrm{quantified} \mathrm{with} \mathrm{ImageJ} \mathrm{software} \mathrm{(right).} \mathrm{Red} \mathrm{bars} \mathrm{represent} \mathrm{the} \mathrm{Median.} \mathrm{**} \mathrm{<} \mathrm{p} 0.01$ compared to NOX4floxp $+/+$. (For interpretation of the references to colour in this figure legend, the reader is referred to the Web version of this article.)

or a mitogenic mixture containing FBS, Insulin, Hydrocortisone and Epidermal Growth Factor (Supplementary Fig. 4A). These differences disappeared at $48 \mathrm{~h}$ after $\mathrm{PH}$, where the response was even higher in the WT hepatocytes (Supplementary Fig. 4B). The increased Myc mRNA levels in response to mitogenic stimuli observed in NOX4-/- hepatocytes $30 \mathrm{~h}$ after $\mathrm{PH}$ correlated with relevant differences in c-Myc protein levels and higher increase in phospho-ERKs and phospho-Akt (Supplementary Fig. 4C).
All these results suggest that acceleration of liver regeneration in NOX4hepKO mice correlates with higher expression of Myc at mRNA and protein levels.

3.4. RNA-seq analysis revealed significant transcriptomic changes in NOX4-/-versus WT mice after PH

In order to better understand the molecular mechanisms that would 
justify the acceleration of liver regeneration and the differences in Myc expression found in NOX4 deleted mice, we performed RNA-seq analysis of liver samples from WT and NOX4-/- mice at $6 \mathrm{~h}, 24 \mathrm{~h}, 48 \mathrm{~h}$ and $168 \mathrm{~h}$ after PH. Results revealed significant differences in gene expression between WT and NOX4-/-, particularly at $24 \mathrm{~h}$ after PH (Fig. 4A). The Gene Ontology (GO) enrichment analysis of the differentially expressed (DE) genes at $24 \mathrm{~h}$ showed that one of the significantly enriched pathways is "response to oxygen-containing compound", of which one of the genes involved is Nox4. The network representation of the top100 DE genes (based on adjusted p-value) that belong to the aforementioned pathway (Supplementary Fig. 5) indicated a central role for Myc, whose expression appeared up-regulated at $6 \mathrm{~h}$ after PH in both WT and NOX4-/- livers, but at $24 \mathrm{~h}$ after PH was maintained at high levels only in NOX4-/- livers, whereas in WT livers decreased at basal levels (Fig. 4B). This differential expression pattern at $24 \mathrm{~h}$ was also observed in other relevant proliferation-related genes that appeared in the enriched oxygen-response network connected to Myc, such as Egr1, Sox9, Jund and Fosb, among others (Fig. 4B and Supplementary Fig. 5). Looking for mitogenic factors whose expression revealed changes, we only found a significant difference in the expression of a member of the Epidermal Growth Factor Receptor (EGFR) family, the Heparin Binding EGF-like Growth Factor (Hbegf gene), whose expression was significantly higher at both 6 and $24 \mathrm{~h}$ after PH in NOX4-/- mice (Fig. 4C). No significant differences were observed in the expression of other members of the EGFR family or in the Met/Hgf (Hepatocyte Growth Factor) pathway. Interestingly, the top $100 \mathrm{DE}$ genes from the enriched oxygenresponse network revealed an axis Nox $4-\operatorname{Src}-$ Myc. In fact, Src expression significantly increased at $24 \mathrm{~h}$ after $\mathrm{PH}$ in NOX4-/- mice, whereas in WT mice no differences were observed (Fig. 4C).

Among the potential processes that regulate hepatocyte proliferation, the TGF- $\beta$ pathway is one of the most significant negative regulators of the expression of $M y c$ [28]. Coincident with the transcriptional changes observed at $24 \mathrm{~h}$ after $\mathrm{PH}$ in NOX4-/- mice, we observed differences in the expression of the Tgfb gene family. Tgfb1, the main isoform expressed in the liver, showed a significant decrease in its expression at $24 \mathrm{~h}$, when compared to WT mice (Fig. 4D). In contrast, $\mathrm{Tg} f \mathrm{~b} 2$ showed increased expression, although in absolute terms, the level of expression is much lower than that of $T g f b 1$. Expression of $T g f b 3$ did
A

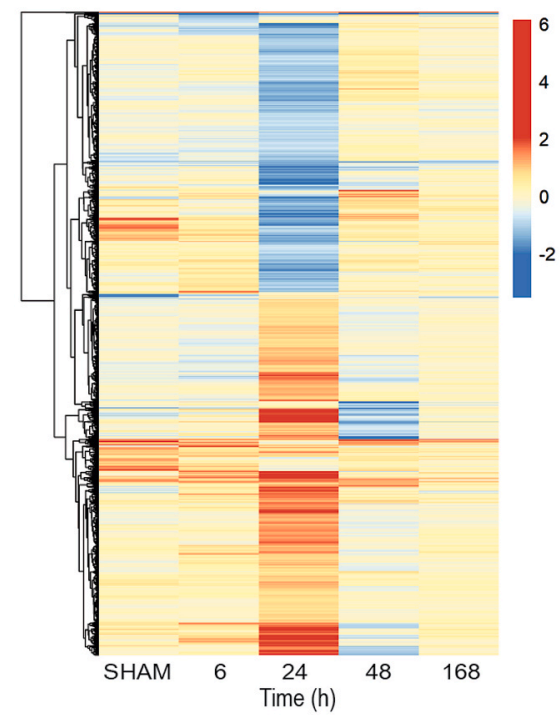

C

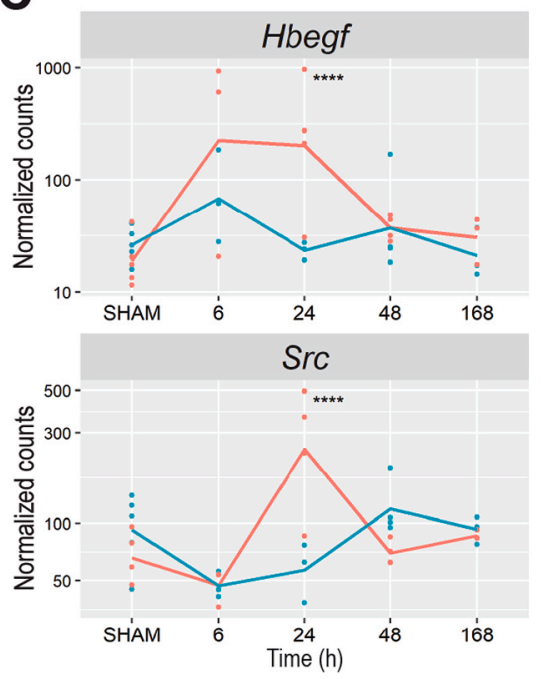

B
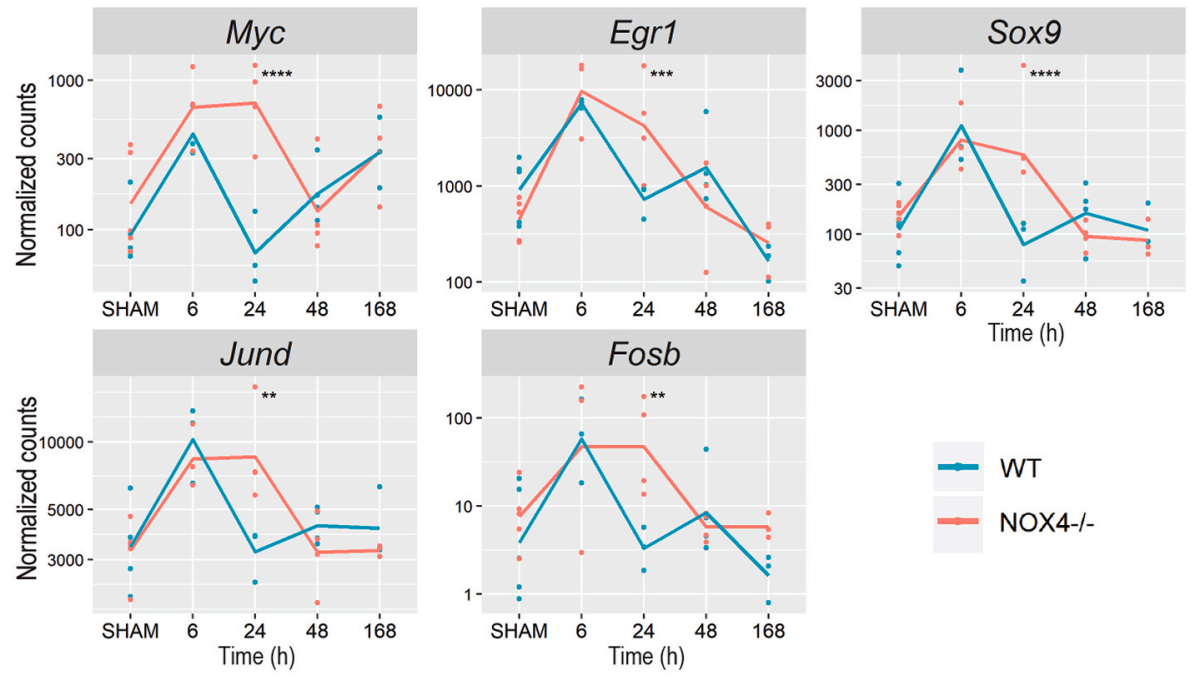

D

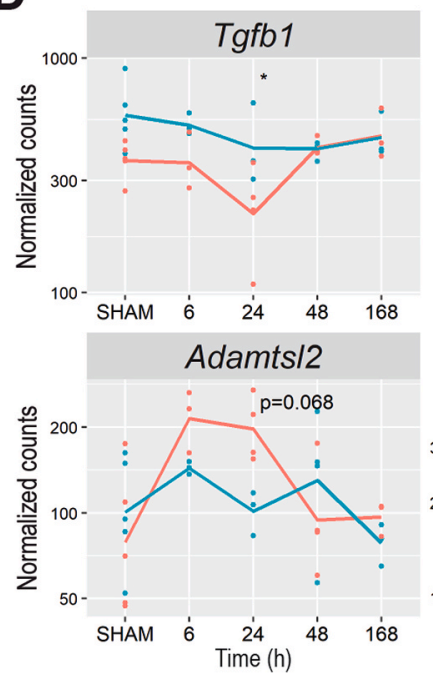

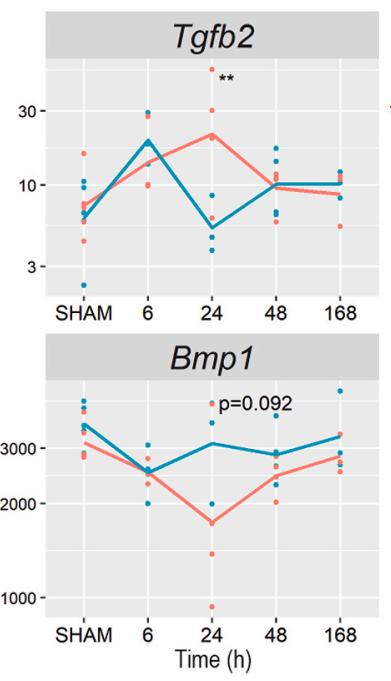
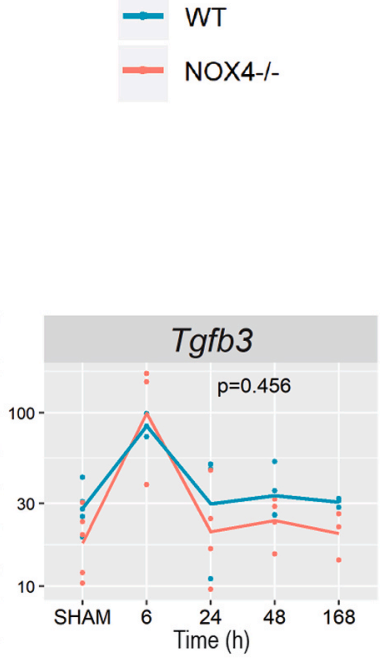

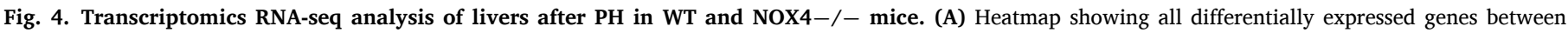

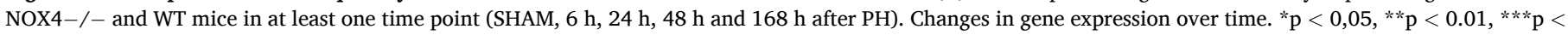

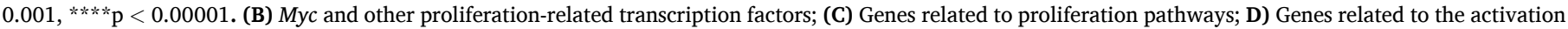
of the TGF- $\beta$ pathway. 
not show significant differences. Furthermore, we focused our attention into two genes that codify proteins whose function is related to the activation of the latent form of TGF- $\beta 1$. Adamtsl2 (a disintegrin and metalloproteinase with thrombospondin repeats-like 2), which interacts with latent TGF- $\beta$ binding protein 1 (LTBP1) and anchors it in the extracellular matrix [29], showed a differential pattern of expression almost significant (adjusted p-value $=0.068$ ) in WT versus NOX4-/mice. Although its expression increased in WT at $6 \mathrm{~h}$ to decrease at $24 \mathrm{~h}$, in NOX4-/- mice, the increase at $6 \mathrm{~h}$ was higher than in WT mice and its expression was maintained high at $24 \mathrm{~h}$ (Fig. 4D). Additionally, the

A

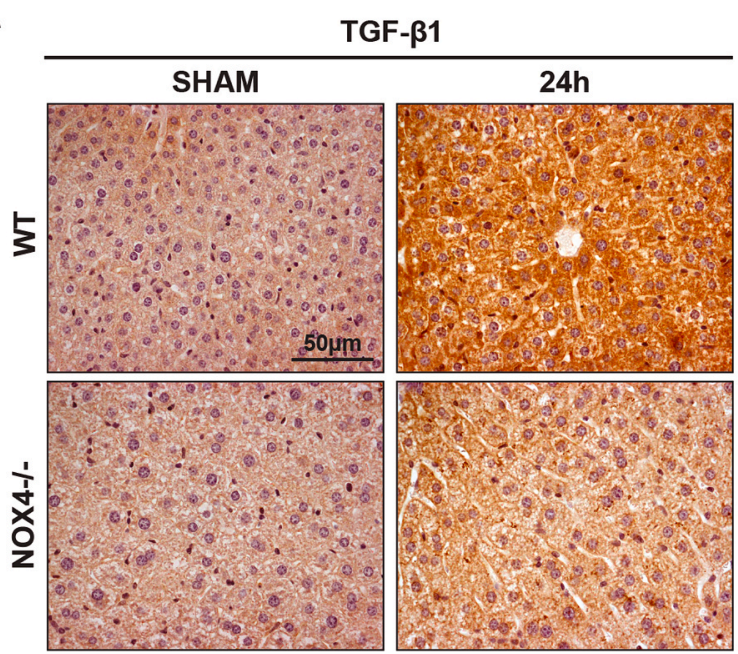

C

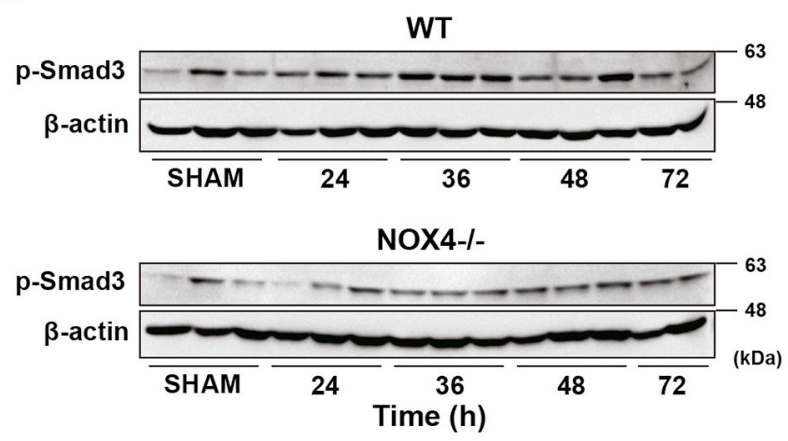

E

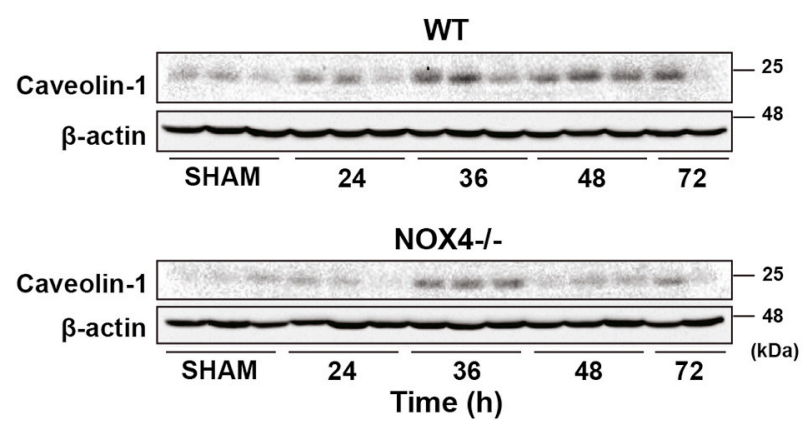

expression of Bmp1, which cleaves LTBPs inducing TGF- $\beta 1$ activation [30], was down-regulated at $6 \mathrm{~h}$ after PH in both WT and NOX4-/mice but, whereas in WT mice the levels returned to basal at $24 \mathrm{~h}$, in NOX4-/- mice continued down-regulated even at lower levels at $24 \mathrm{~h}$ after PH (Fig. 4D). These results indicated that the TGF- $\beta$ pathway could be differentially activated along the time in WT and NOX4-/- mice.

B

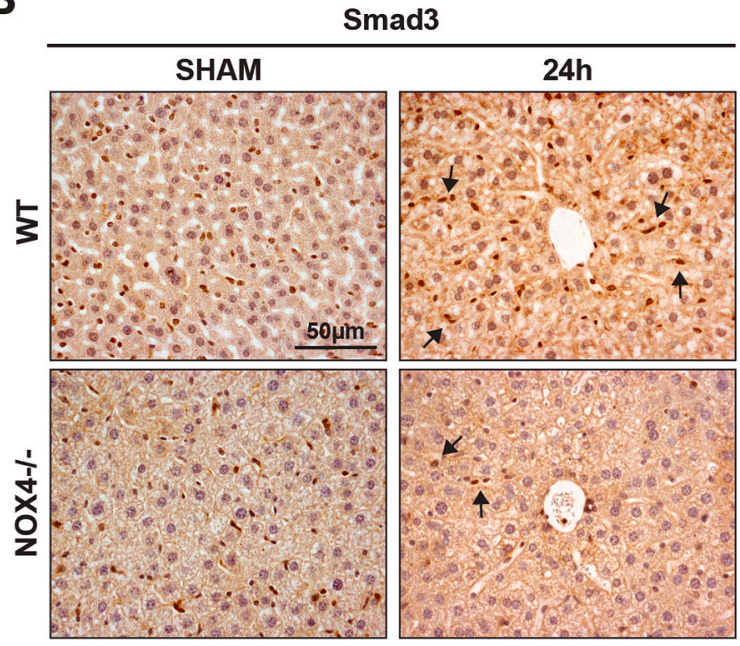

D
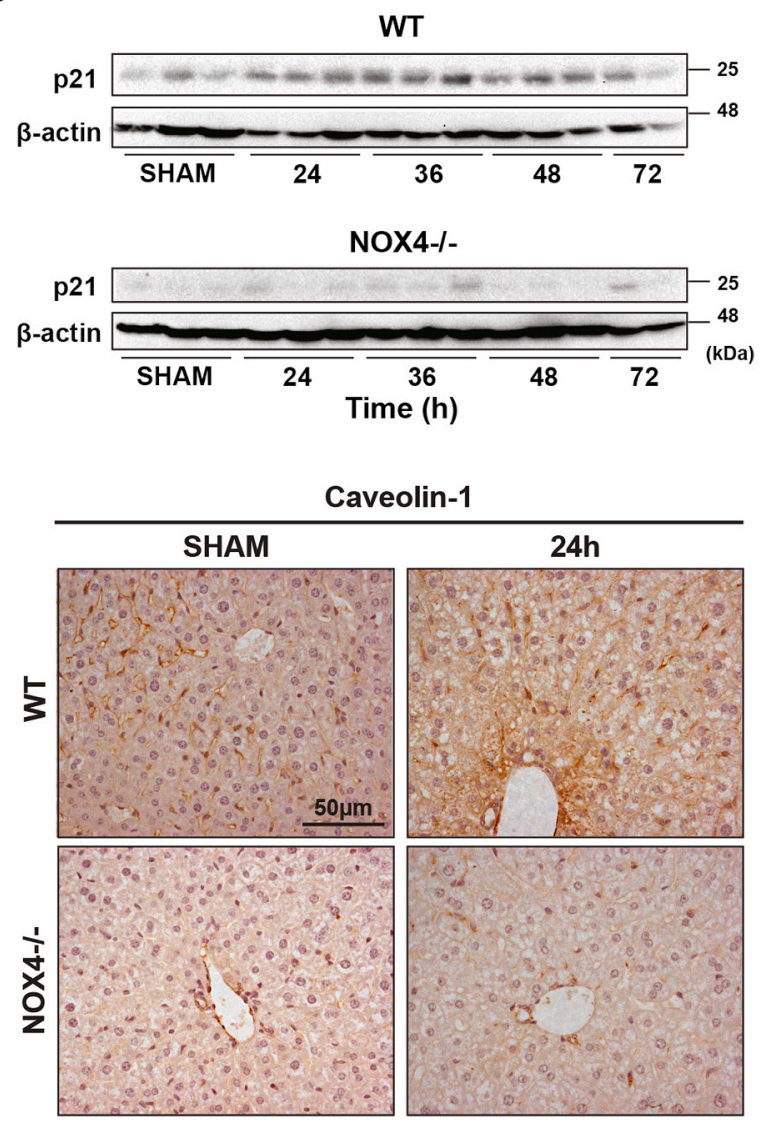

Fig. 5. Analysis of the TGF- $\beta$ pathway in WT and NOX4-/- livers after PH. (A) TGF- $\beta$ and (B) Smad3 immunostaining was performed in WT and NOX4-/mice. Arrows indicate Smad3 positive nuclei. Analysis of p-Smad3 (C), p21 (D) and caveolin-1 (E) by Western Blot (left). $\beta$-actin was used as a loading control. In (E), on right, caveolin-1 immunostaining. 
3.5. After PH, Nox4-/- and NOX4hepKO livers showed an earlier and increased attenuation of the TGF- $\beta$ pathway

IHC analysis of the mature, active, form of TGF- $\beta 1$ revealed that TGF$\beta 1$ staining considerably increased in WT liver tissues, but this increase was significantly lower in NOX4-/- and barely observed in NOX4HepKO at 24 and $36 \mathrm{~h}$ after $\mathrm{PH}$, respectively (Fig. 5A and Supplementary Fig. 6A, respectively). This correlated with lower number of Smad3 positive nuclei in NOX4-/- and NOX4HepKO, analyzed by IHC in liver tissues (Fig. 5B and Supplementary Fig. 6B). Western blot analysis revealed an increase in the levels of phospho-Smad3 after PH in both WT mice that was delayed and attenuated in NOX4-/- and NOX4HepKO mice (Fig. 5C and Supplementary Fig. 6C), correlating with decreased protein levels of one of its more relevant targets, p21, a cyclin-CDK inhibitor (Fig. 5D). Previous results had demonstrated that deficiency in the liver of caveolin-1, a protein that is located in lipid rafts at the cell membrane and involved in intracellular trafficking of receptors, impairs the TGF- $\beta$ pathway and improves liver regeneration [31]. Interestingly, concomitant with the alteration in the activation of the TGF- $\beta$ pathway, we found lower expression of caveolin-1 in NOX4-/- mice when compared to WT mice (Fig. 5E).

Worthy to note that primary isolated hepatocytes from WT and NOX4-/- mice demonstrated a correct response to exogenous TGF- $\beta 1$ inhibiting FBS-induced increase in cyclins expression and hepatocyte proliferation, analyzed as cell number or Ki67+ cells, regardless Nox4 is expressed or not (Fig. 6A-C). Similar data were observed in primary hepatocytes from NOX4hepKO mice (results not shown). Levels of Smad2 phosphorylation in response to TGF- $\beta 1$ were similar in WT and
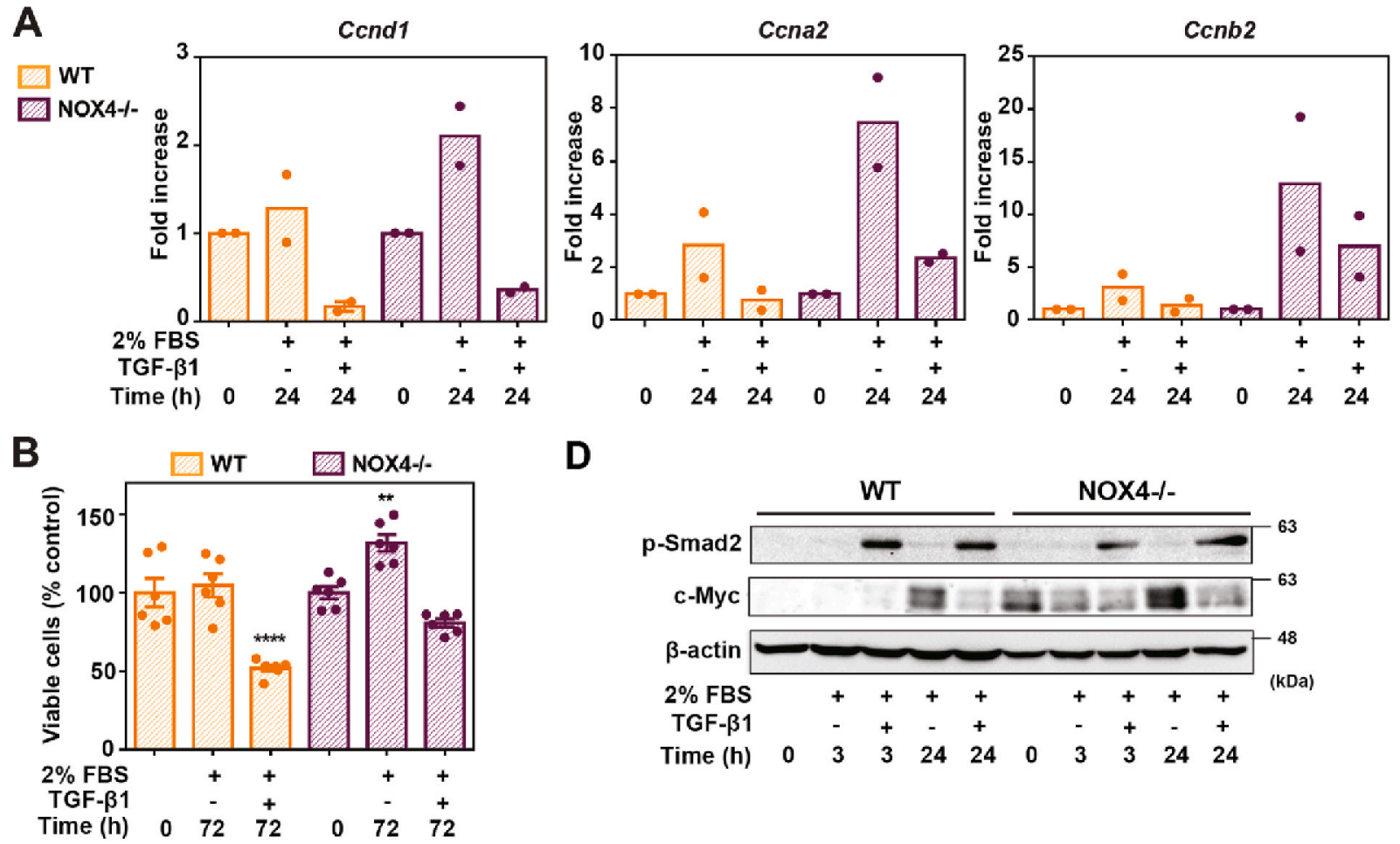

D

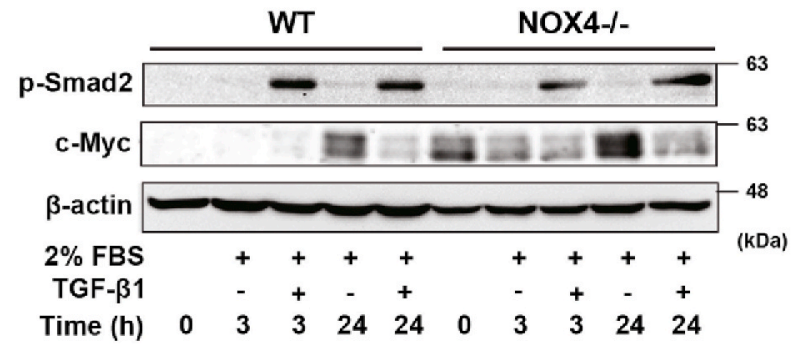

C

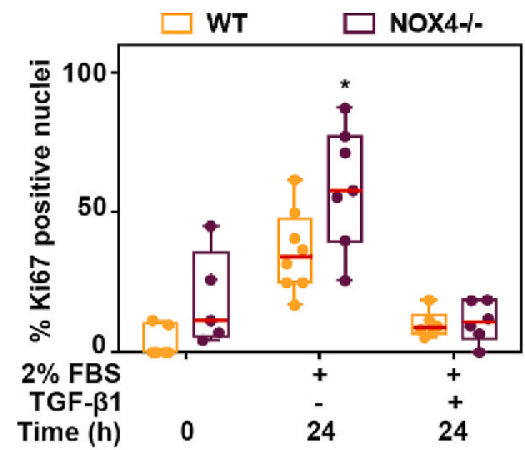

E

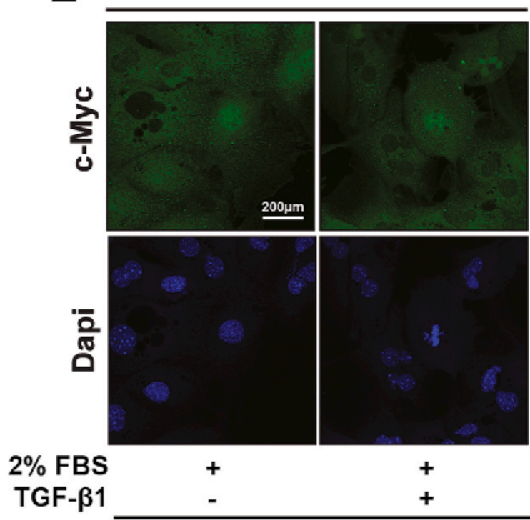

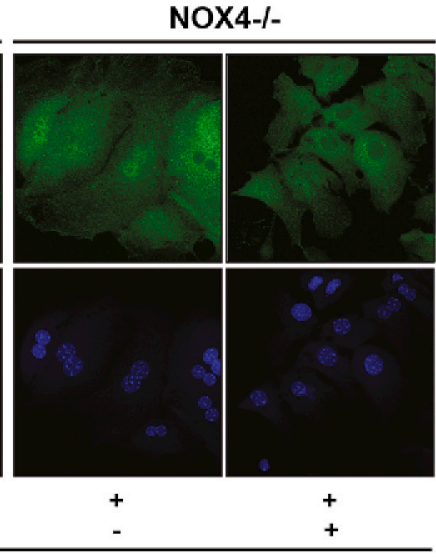

24h

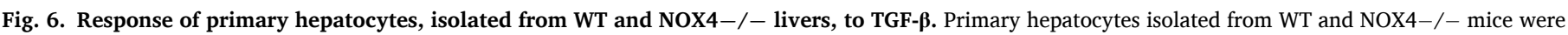

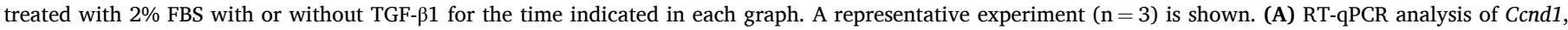

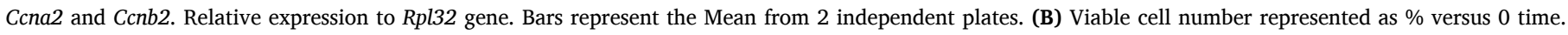

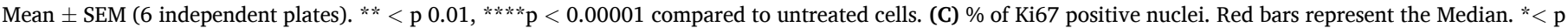

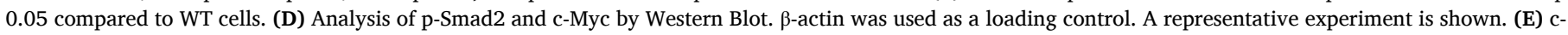

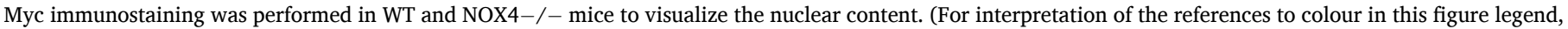
the reader is referred to the Web version of this article.) 
NOX4-/- hepatocytes (Fig. 6D). c-Myc levels were higher at basal levels, as well as in response to FBS, in NOX4-/- hepatocytes, but TGF$\beta 1$ showed identical capacity in inhibiting its up-regulation in WT and in NOX4-/- hepatocytes (Fig. 6D-E). These results suggest that Nox4 is not required for TGF- $\beta 1$-induced growth inhibition of cell cycle.

Altogether, in vivo results suggest that deletion of Nox4 in mice accelerates liver regeneration, which correlates with changes in the transcriptional program at $24 \mathrm{~h}$ after $\mathrm{PH}$, increased expression of $M y c$ and other mitogenic-related genes and attenuation in the activation of the TGF- $\beta$ pathway (Fig. 7 , left). In vitro results indicate that hepatocytes from Nox4 deleted mice show increased proliferative capacity, coincident with higher $M y c$ and cyclins expression, in response to FBS or mitogenic signals, but they maintain the capacity to respond to TGF- $\beta 1$ in terms of growth inhibition (Fig. 7, right).

\section{Discussion}

Liver regeneration is a complex process that, despite extensive study, is not completely understood. Numerous signaling pathways, as well as interactions among different cell types, make the process extremely attractive to glean molecular insights into the self-renewal of mature cells, a property frequently associated with stem cells. Hepatocytes appear to be cell autonomous in deciding their replication fate, which depends on reprograming different molecular events in different cell types [32]. Results here support a role for Nox4 in negatively regulating liver regeneration. Interestingly, similar results were observed in NOX4-/- and NOX4HepKO models, which indicates that the effect on liver regeneration is mostly due to Nox4 function in hepatocytes. This is not surprising, since Nox4 expression is two orders of magnitude higher in hepatocytes than in other liver cell types, such as stellate cells, and even one order of magnitude higher than the expression in myofibroblasts, under fibrotic conditions [6]. In fact, residual Nox4 expression in the livers of NOX4HepKO mice was barely detectable, lower than $0.5 \%$ when compared to the corresponding WT mice (results not shown). Nox4 expression is down-regulated after PH in mice, which is probably necessary for an efficient hepatocyte proliferation, due to its mitoinhibitory role [10]. Down-regulation of Nox4 might occur by the increase in proliferative signals, since both EGF and HGF inhibit its expression [33,34]. In the experimental animal models used in this study, where Nox4 expression is deleted, hepatocytes were more "primed" to respond to mitogenic signals in a fastest way. An interesting aspect to be mentioned is that although a slight increase in the liver to body weight ratio was observed in NOX4-/- mice versus WT, long-term analysis revealed that proliferation was arrested, and the liver reached a size that did not overcome the initial one. Many signals contribute to the termination of liver regeneration and maintenance of standard liver mass [15] and Nox4 does not appear to be essential for this process.

Trying to analyze the potential mechanism that could be regulated by Nox4, we found a stronger activation of the c-Myc pathway in vivo, as well as in hepatocytes in culture. c-Myc is a relevant regulator of the expression of cell-cycle related genes [35] and it has been suggested that hepatocytes require up-regulation of $M y c$ after $\mathrm{PH}$ to efficiently exit $\mathrm{G}_{0}$ [27]. Interestingly, c-Myc not only regulates hepatocyte proliferation, but also cell size [27]. Here, the increase in Myc expression in Nox4 deleted mice correlated with a significant increase in hepatocyte cell size. It is well known that hypertrophy without cell division precedes proliferation after $2 / 3 \mathrm{PH}$ and almost equally contribute to regeneration [36]. The increase in cell size observed at $24-36 \mathrm{~h}$ after PH in both mouse models of Nox4 deletion correlated with the peak of Myc expression and preceded the peak of cell proliferation. Furthermore, c-Myc could also contribute to maintain lipid homeostasis, through

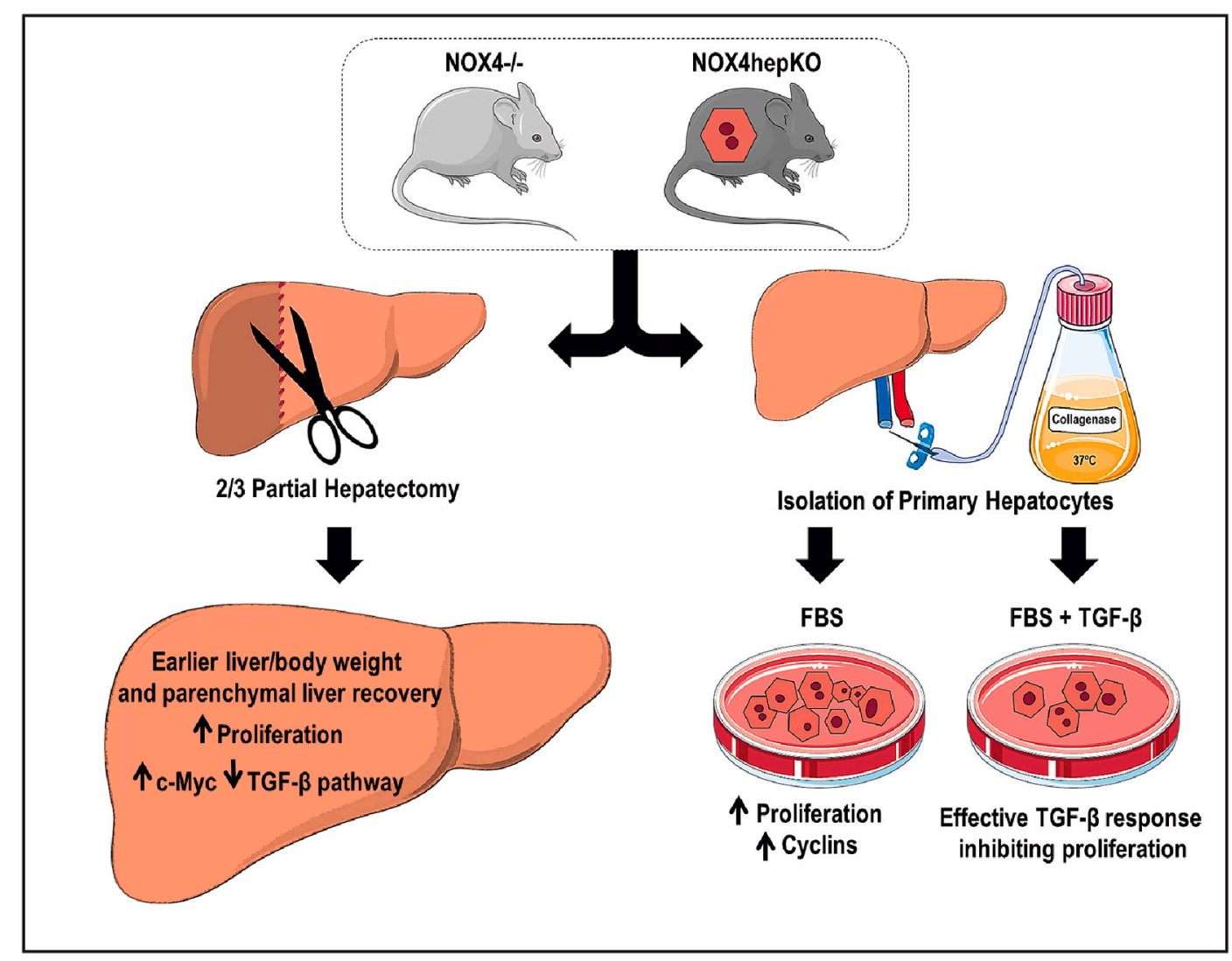

Fig. 7. Summary diagram. Left: After partial hepatectomy, Nox4 deleted mice show earlier liver/body weight recovery, concomitant with higher proliferation, upregulation of Myc and down-regulation of the TGF- $\beta$ pathway. Right: Primary hepatocytes from Nox4 deleted mice show higher proliferation in response to FBS, while maintaining TGF- $\beta$-induced growth inhibitory response. 
activating fatty acid oxidation for energy demands [37]. Indeed, overactivation of c-Myc could also explain the faster recovery of regenerative liver steatosis observed in NOX4-/- mice.

RNA-seq analysis revealed significant differences at the transcriptional level after PH in NOX4-/- mice when compared to WT mice. The network of the top100 differentially expressed genes at $24 \mathrm{~h}$ from the "response to oxygen-containing compound" pathway located Myc in a node of proliferation-related genes whose expression decays at $24 \mathrm{~h}$ after $\mathrm{PH}$ in WT mice, but it is maintained, or even enhanced, at this same time in NOX4-/- mice. Significant increase in the expression of Hbegf, a member of the EGFR ligands family previously shown to have potent protective and mitogenic effects in liver regeneration [38], was observed specifically at $24 \mathrm{~h}$ after $\mathrm{PH}$ in NOX4-/- mice, as well as the expression of $\mathrm{Src}$, a member of the cytosolic family of tyrosine kinase proteins.

Different evidences support a role for TGF- $\beta$ inhibiting $M y c$ expression [28]. In hepatocytes, TGF- $\beta 1$ inhibits growth and induces apoptosis in hepatocytes $[39,40]$ and one of its earlier effects is down-regulation of the mitogen-induced Myc early expression [41], without an effect on the expression of other proto-oncogenes, such as Hras. Here, we found that acceleration of liver regeneration and increased expression of $M y c$ in both mice models of Nox 4 deletion could be attributed to the attenuation of the TGF- $\beta$ pathway. It was proposed that transient escape of regenerative hepatocytes from TGF- $\beta$-induced growth inhibition and apoptosis is achieved through decrease in the expression of TGF- $\beta$ receptors [42] and the acquisition of survival signals [43]. Nevertheless, a mitoinhibitory response to TGF- $\beta$ is still present in regenerating hepatocytes, since intravenous TGF- $\beta 1 / 2$ reversibly inhibits the proliferative response of the liver to $\mathrm{PH}$ [44] and inactivation of the TGF- $\beta$ pathway results in an increased proliferative response after PH [45]. Anti-TGF- $\beta$ molecular intervention also facilitates liver regeneration upon acute dimethylnitrosamine (DMN) or CCl4 induced injury [46,47]. Results here indicate that in the absence of Nox 4 expression, the TGF- $\beta$ pathway was attenuated in hepatocytes, which could contribute to the acceleration of the liver regeneration.

Although it is well known that TGF- $\beta$ up-regulates NOX4 in stellate cells and hepatocytes $[6,7,9]$, it is less well recognized that NOX4 can reciprocally regulate TGF- $\beta$ /SMAD signaling. The RNA-seq experiment revealed the potential mechanism responsible for this effect, which appears to be indirect, related to other cell-cell interactions and effects on extracellular matrix. On one side, TGF- $\beta$ ligands, which are expressed in non-parenchymal liver cells, showed differences between WT and NOX4-/- mice. Tgfb1 expression was significantly lower at $24 \mathrm{~h}$ after PH in NOX4-/- mice when compared to WT mice. Another member of the family, $T g f b 2$, by contrast, showed higher levels but its expression was much lower than that of Tgfb1. TGF- $\beta 2$ up-regulation correlates with fibrotic markers and play a prominent role in biliary liver diseases [48, 49], but its expression during liver regeneration is 10 times lower than that of TGF- $\beta 1$ [50]. Considering that Smad3 phosphorylation was clearly decreased in Nox4 deleted mice, the decrease in $T g f b 1$ expression appears to have a higher impact that the increase in $T g f b 2$ expression. Furthermore, activation of the latent TGF- $\beta 1$ at the extracellular matrix may be affected by the absence of Nox4. In support of this hypothesis, Adamtsl2, which interacts with latent LTBP1 and anchors it to the extracellular matrix, showed an increase in its expression in NOX4-/mice at $24 \mathrm{~h}$ after PH. Mutations in ADAMTSL2 have been described in human diseases and lead to dysregulation of TGF- $\beta$ signaling correlating with increase in active TGF- $\beta 1$ [29]. Furthermore, the expression of $B m p 1$, a metalloproteinase that cleaves LTBPs releasing the active form of TGF- $\beta 1$ [30], is decreased at $24 \mathrm{~h}$ after PH in NOX4-/- mice. Altogether, these results reveal a mutual regulation between Nox4 and the TGF- $\beta$ pathway: TGF- $\beta 1$ is the main up-regulator of Nox 4 expression and Nox 4 could contribute to modulate the process of TGF- $\beta 1$ activation. A fine down-regulation of Nox4 expression during the first hours after PH may be necessary for an efficient hepatocyte proliferation and liver regeneration.
Despite these in vivo observations, in vitro experiments in NOX4-/hepatocytes indicated that they continue to respond to TGF- $\beta 1$ in terms of growth inhibition, Smad2 phosphorylation or down-regulation of $M y c$ levels, maintaining its suppressor function. Indeed, Nox4 deletion increases the hepatocyte mitogenic response and modify the process of TGF- $\beta 1$ activation in vivo, which increases the fastest and more efficient response of hepatocytes to mitogenic signals during liver regeneration. But the mechanism of response to extracellular TGF- $\beta 1$ is not altered. NOX4 does not appear to be required for TGF- $\beta 1$-mediated Myc downregulation, which was proposed to be a SMAD-4 dependent mechanism [51]. This could explain the arrest of proliferation in vivo long time after $\mathrm{PH}$, when the expression/activation of TGF- $\beta 1$, or other members of the family, increase.

\section{Conclusions}

NOX4 has been proposed as a potential therapeutic target in human liver chronic diseases [8], due to its role mediating TGF- $\beta$ actions in liver fibrosis [5]. Results presented here would support the potential benefit of its inhibition also to favor liver regeneration from the remaining healthy hepatocytes. Nox4 does not appear to be essential for the termination of liver regeneration, which reinforces its potential as therapeutic target, without adverse effects. Nevertheless, considering that inhibiting NOX4 could be detrimental under preneoplastic conditions, NOX4 inhibition must be considered at early stages of chronic inflammation and fibrosis in the liver.

\section{Author contributions}

I.F. and N.J.T. conceived and designed the study.

M.H.-I. acquired data, with the participation in some experiments of J.L.-L., E.C.-M., D.C.-D., E.G.-S and J.X.J.

M.G performed the RNA-seq.

B.M.-M. and A.E.-C. analyzed and interpreted the RNA-seq data.

M.H.-I., J.L.-L. and I.F. analyzed and interpreted data and drafted the article, which was revised it critically for important intellectual content by E.G.-S, N.J.T. and V.J.

All authors participated in manuscript revision and final approval of the version to be submitted.

\section{Funding}

This work was supported by grants from: 1) Agencia Estatal de Investigación and Instituto de Salud Carlos III, Ministerio de Ciencia e Innovación (MICINN), Spain, cofounded by FEDER funds/European Regional Development Fund - a way to build Europe- (SAF2015-64149R and RTI2018-094079-B-I00 to I.F.; PT17/0009/0019 to A.E.-C.; Programa Operativo FEDER: Plurirregional de España (POPE) 2014-2020 and Catalunya 2014-2020 to CNAG-CRG authors; 2) NIH 2R01DK083283 (to N.J.T.). M.H.-I. was recipient of a predoctoral grant from IDIBELL-Oncobell Program. D.C-D. was recipient of a pre-doctoral grant from the FPI program. J.L-L. was recipient of Boehringer Ingelheim Fonds Travel grant. The CIBEREHD, National Biomedical Research Institute on Liver and Gastrointestinal Diseases, is funded by the Instituto de Salud Carlos III, Spain. We thank the Generalitat de Catalunya through the CERCA Programme and the Departament de Salut and Departament d'Empresa i Coneixement, the Centro de Excelencia Severo Ochoa and the MICINN to the EMBL partnership for institutional support.

\section{Declaration of competing interest}

The authors declare that they have no known competing financial interests or personal relationships that could have appeared to influence the work reported in this paper. 


\section{Acknowledgements}

We acknowledge the continuous help and advices of Esther Bertran (IDIBELL, TGF-beta \& Cancer group). We acknowledge technical support of Benjamín Torrejón (Scientific and Technical Services, University of Barcelona, CCiTUB), Lola Mulero (Center of Regenerative Medicine in Barcelona CMR[B]) and Rosa Bonavia and Pilar Rosario Sánchez (IDIBELL Animal Facilities). We also thank Sarah Fish, Ali Dehnad and Suvarthi Das for their help in Natalie Török's laboratory at UC Davis. Authors are also grateful to the ERASMUS+ student Aleksandra Murzyn for support in some experimental procedures. We thank Karl HeinzKrause and Michelangelo Foti and their teams (University of Geneva) for technical advices and helpful discussions. Finally, I.F. acknowledges Profs. Nelson Fausto and Hyam L. Leffert for encouraging discussions about molecular mechanisms regulating liver regeneration.

\section{Appendix A. Supplementary data}

Supplementary data to this article can be found online at https://doi. org/10.1016/j.redox.2020.101841.

\section{References}

[1] H. Buvelot, V. Jaquet, K.H. Krause, Mammalian NADPH oxidases, Methods Mol. Biol. 1982 (2019) 17-36.

[2] K.D. Martyn, L.M. Frederick, K. von Loehneysen, M.C. Dinauer, U.G. Knaus, Functional analysis of Nox4 reveals unique characteristics compared to other NADPH oxidases, Cell. Signal. 18 (1) (2006) 69-82.

[3] L. Serrander, L. Cartier, K. Bedard, B. Banfi, B. Lardy, O. Plastre, et al., NOX4 activity is determined by mRNA levels and reveals a unique pattern of ROS generation, Biochem. J. 406 (1) (2007) 105-114.

[4] J.X. Jiang, N. Török, NADPH oxidases in chronic liver diseases, Adv Hepatol 2014 (2014) 1-8.

[5] E. Crosas-Molist, E. Bertran, I. Fabregat, Cross-talk between TGF- $\beta$ and NADPH oxidases during liver fibrosis and hepatocarcinogenesis, Curr. Pharmaceut. Des. 21 (41) (2015) 5964-5976.

[6] P. Sancho, J. Mainez, E. Crosas-Molist, C. Roncero, C.M. Fernández-Rodriguez, F. Pinedo, et al., NADPH oxidase NOX4 mediates stellate cell activation and hepatocyte cell death during liver fibrosis development, Plos One 7 (9) (2012), e45285.

[7] J.X. Jiang, X. Chen, N. Serizawa, C. Szyndralewiez, P. Page, K. Schröder, et al, Liver fibrosis and hepatocyte apoptosis are attenuated by GKT137831, a novel NOX4/NOX1 inhibitor in vivo, Free Radic. Biol. Med. 53 (2) (2012) 289-296.

[8] E. Crosas-Molist, I. Fabregat, Role of NADPH oxidases in the redox biology of liver fibrosis, Redox Biol. 6 (2015) 106-111.

[9] I. Carmona-Cuenca, C. Roncero, P. Sancho, L. Caja, N. Fausto, M. Fernández, et al., Upregulation of the NADPH oxidase NOX4 by TGF-beta in hepatocytes is required for its pro-apoptotic activity, J. Hepatol. 49 (6) (2008) 965-976.

[10] E. Crosas-Molist, E. Bertran, P. Sancho, J. López-Luque, J. Fernando, A. Sánchez, et al., The NADPH oxidase NOX4 inhibits hepatocyte proliferation and liver cancer progression, Free Radic. Biol. Med. 69 (2014) 338-347.

[11] E. Crosas-Molist, E. Bertran, I. Rodriguez-Hernandez, C. Herraiz, G. Cantelli, A. Fabra, et al., The NADPH oxidase NOX4 represses epithelial to amoeboid transition and efficient tumour dissemination, Oncogene 36 (21) (2017) 3002-3014.

[12] J. López-Luque, I. Fabregat, Revisiting the liver: from development to regeneration - what we ought to know!, Int. J. Dev. Biol. 62 (6-7-8) (2018) 441-451.

[13] N. Fausto, J.S. Campbell, K.J. Riehle, Liver regeneration, Hepatology 43 (S1) (2006) S45-53.

[14] G.K. Michalopoulos, Advances in liver regeneration, Expet Rev. Gastroenterol. Hepatol. 8 (8) (2014) 897-907.

[15] G.K. Michalopoulos, Hepatostat: liver regeneration and normal liver tissue maintenance, Hepatology 65 (4) (2017) 1384-1392.

[16] G.K. Michalopoulos, Liver regeneration after partial hepatectomy, Am. J. Pathol. 176 (1) (2010) 2-13.

[17] S. Carnesecchi, C. Deffert, Y. Donati, O. Basset, B. Hinz, O. Preynat-Seauve, et al., A key role for NOX4 in epithelial cell death during development of lung fibrosis, Antioxidants Redox Signal. 15 (3) (2011) 607-619.

[18] A. Bettaieb, J.X. Jiang, Y. Sasaki, T.-I. Chao, Z. Kiss, X. Chen, et al., Hepatocyte nicotinamide adenine dinucleotide phosphate reduced oxidase 4 regulates stress signaling, fibrosis, and Insulin sensitivity during development of steatohepatitis in mice, Gastroenterology 149 (2) (2015) 468-480, e10.

[19] G.M. Higgins, R.M. Anderson, Experimental pathology of liver: restoration of liver of white rat following partial surgical removal, Arch. Pathol. 12 (1931) 186-202.

[20] E. Gonzalez-Sanchez, D. Firrincieli, C. Housset, N. Chignard, Expression patterns of nuclear receptors in parenchymal and non-parenchymal mouse liver cells and their modulation in cholestasis, Biochim. Biophys. Acta (BBA) - Mol. Basis Dis. 1863 (7) (2017) 1699-1708.
[21] J. López-Luque, D. Caballero-Díaz, A. Martinez-Palacián, C. Roncero, J. MorenoCàceres, M. García-Bravo, et al., Dissecting the role of epidermal growth factor receptor catalytic activity during liver regeneration and hepatocarcinogenesis, Hepatology 63 (2) (2016) 604-619.

[22] J. López-Luque, E. Bertran, E. Crosas-Molist, O. Maiques, A. Malfettone, L. Caja, et al., Downregulation of epidermal growth factor receptor in hepatocellular carcinoma facilitates transforming growth factor- $\beta$-induced epithelial to amoeboid transition, Canc. Lett. 464 (2019) 15-24.

[23] A. Dobin, C.A. Davis, F. Schlesinger, J. Drenkow, C. Zaleski, S. Jha, et al., STAR: ultrafast universal RNA-seq aligner, Bioinformatics 29 (1) (2013) 15-21.

[24] B. Li, C.N. Dewey, RSEM: accurate transcript quantification from RNA-Seq data with or without a reference genome, BMC Bioinf. 12 (1) (2011) 323.

[25] M.I. Love, W. Huber, S. Anders, Moderated estimation of fold change and dispersion for RNA-seq data with DESeq2, Genome Biol. 15 (12) (2014) 550.

[26] J. Reimand, M. Kull, H. Peterson, J. Hansen, J. g Vilo, Profiler-a web-based toolset for functional profiling of gene lists from large-scale experiments, Nucleic Acids Res. 35 (suppl_2) (2007) W193-200.

[27] E. Baena, A. Gandarillas, M. Vallespinos, J. Zanet, O. Bachs, C. Redondo, et al., cMyc regulates cell size and ploidy but is not essential for postnatal proliferation in liver, Proc. Natl. Acad. Sci. Unit. States Am. 102 (20) (2005) 7286-7291.

[28] J.P. Frederick, N.T. Liberati, D.S. Waddell, Y. Shi, X.-F. Wang, Transforming growth factor -mediated transcriptional repression of c-myc is dependent on direct binding of Smad3 to a novel repressive Smad binding element, Mol. Cell Biol. 24 (6) (2004) 2546-2559.

[29] C.L. Goff, F. Morice-Picard, N. Dagoneau, L.W. Wang, Y.J. Crow, F. Bauer, et al., ADAMTSL2 mutations in geleophysic dysplasia demonstrate a role for ADAMTSlike proteins in TGF- $\beta$ bioavailability regulation, Nat. Genet. 40 (9) (2008) 1119-1123.

[30] G. Ge, D.S. Greenspan, BMP1 controls TGF 1 activation via cleavage of latent TGFß-binding protein, J. Cell Biol. 175 (1) (2006) 111-120.

[31] R. Mayoral, L. Bosca, P. Martín-Sanz, Impairment of transforming growth factor beta signaling in caveolin-1-deficient hepatocytes: role in liver regeneration, J. Biol. Chem. 285 (6) (2010) 3633-3642.

[32] N. Fausto, J.S. Campbell, K.J. Riehle, Liver regeneration, J. Hepatol. 57 (3) (2012) 692-694.

[33] I. Carmona-Cuenca, B. Herrera, J.-J. Ventura, C. Roncero, M. Fernández, I. Fabregat, EGF blocks NADPH oxidase activation by TGF- $\beta$ in fetal rat hepatocytes, impairing oxidative stress, and cell death, J. Cell. Physiol. 207 (2) (2006) 322-330.

[34] Z. Cao, T. Ye, Y. Sun, G. Ji, K. Shido, Y. Chen, et al., Targeting the vascular and perivascular niches as a regenerative therapy for lung and liver fibrosis, Sci. Transl. Med. 9 (405) (2017), eaai8710.

[35] G. Bretones, M.D. Delgado, J. León, Myc and cell cycle control, Biochim. Biophys. Acta Gene Regul. Mech. 1849 (5) (2015) 506-516.

[36] Y. Miyaoka, K. Ebato, H. Kato, S. Arakawa, S. Shimizu, A. Miyajima, Hypertrophy and unconventional cell division of hepatocytes underlie liver regeneration, Curr. Biol. 22 (13) (2012) 1166-1175.

[37] L.R. Edmunds, P.A. Otero, L. Sharma, S. D’Souza, J.M. Dolezal, S. David, et al., Abnormal lipid processing but normal long-term repopulation potential of myc-/hepatocytes, Oncotarget 7 (21) (2016) 30379-30395.

[38] N.C. Khai, T. Takahashi, H. Ushikoshi, S. Nagano, K. Yuge, M. Esaki, et al., In vivo hepatic HB-EGF gene transduction inhibits Fas-induced liver injury and induces liver regeneration in mice: a comparative study to HGF, J. Hepatol. 44 (6) (2006) 1046-1054.

[39] B.I. Carr, I. Hayashi, E.L. Branum, H.L. Moses, Inhibition of DNA synthesis in rat hepatocytes by platelet-derived type $\beta$ transforming growth factor, Canc. Res. 46 (5) (1986) 2330-2334.

[40] A. Sánchez, A.M. Álvarez, M. Benito, I. Fabregat, Apoptosis induced by transforming growth factor- $\beta$ in fetal hepatocyte primary cultures: involvement of reactive oxygen intermediates, J. Biol. Chem. 271 (13) (1996) 7416-7422.

[41] A. Sanchez, A.M. Alvarez, M. Benito, I. Fabregat, Transforming growth factor-beta modulates growth and differentiation of fetal hepatocytes in primary culture, J. Cell. Physiol. 165 (2) (1995) 398-405.

[42] R.S. Chart, D.T. Price, S.R. Sue, W.C. Meyers, R.L. Jirtle, Down-regulation of transforming growth factor beta receptor type I, II, and III during liver regeneration, Am. J. Surg. 169 (1) (1995) 126-132.

[43] B. Herrera, A.M. Álvarez, J. Beltrán, F. Valdés, I. Fabregat, M. Fernández, Resistance to TGF- $\beta$-induced apoptosis in regenerating hepatocytes, J. Cell. Physiol. 201 (3) (2004) 385-392.

[44] W.E. Russell, R.J. Coffey Jr., A.J. Ouellette, H.L. Moses, Type $\beta$ transforming growth factor reversibly inhibits the early proliferative response to partial hepatectomy in the rat, Proc. Natl. Acad. Sci. Unit. States Am. 85 (14) (1988) 5126-5130.

[45] J. Romero-Gallo, E.G. Sozmen, A. Chytil, W.E. Russell, R. Whitehead, W.T. Parks, et al., Inactivation of TGF- $\beta$ signaling in hepatocytes results in an increased proliferative response after partial hepatectomy, Oncogene 24 (18) (2005) 3028-3041.

[46] T. Nakamura, T. Ueno, M. Sakamoto, R. Sakata, T. Torimura, O. Hashimoto, et al., Suppression of transforming growth factor- $\beta$ results in upregulation of transcription of regeneration factors after chronic liver injury, J. Hepatol. 41 (6) (2004) 974-982.

[47] S. Karkampouna, M.-J. Goumans, P. ten Dijke, S. Dooley, M. Kruithof-de Julio, Inhibition of TGF $\beta$ type I receptor activity facilitates liver regeneration upon acute CCl4 intoxication in mice, Arch. Toxicol. 90 (2) (2016) 347-357. 
[48] A. Dropmann, T. Dediulia, K. Breitkopf-Heinlein, H. Korhonen, M. Janicot, S. N. Weber, et al., TGF- $\beta 1$ and TGF- $\beta 2$ abundance in liver diseases of mice and men, Oncotarget 7 (15) (2016) 19499-19518.

[49] A. Dropmann, S. Dooley, B. Dewidar, S. Hammad, T. Dediulia, J. Werle, et al., TGF$\beta 2$ silencing to target biliary-derived liver diseases, Gut 69 (9) (2020) 1677-1690.
[50] S.B. Jakowlew, D. Danielpour, J. Wu, A.B. Roberts, N. Fausto, Transforming growth factor- $\beta$ (TGF- $\beta$ ) isoforms in rat liver regeneration: messenger RNA expression and activation of latent TGF- $\beta$, Cell Regul. 2 (7) (1991) 535-548.

[51] M.J. Calonge, J. Massague, Smad4/DPC4 silencing and hyperactive ras jointly disrupt transforming growth factor-beta antiproliferative responses in colon cancer cells, J. Biol. Chem. 274 (47) (1999) 33637-33643. 